\title{
Geometric singular perturbation analysis of a dynamical target mediated drug disposition model
}

\section{Kristiansen, Kristian Uldall}

Published in:

Journal of Mathematical Biology

Link to article, DOI:

$10.1007 / \mathrm{s} 00285-019-01354-3$

Publication date:

2019

Document Version

Peer reviewed version

Link back to DTU Orbit

Citation (APA):

Kristiansen, K. U. (2019). Geometric singular perturbation analysis of a dynamical target mediated drug

disposition model. Journal of Mathematical Biology, 79(1), 187-222. https://doi.org/10.1007/s00285-019-01354-3

\section{General rights}

Copyright and moral rights for the publications made accessible in the public portal are retained by the authors and/or other copyright owners and it is a condition of accessing publications that users recognise and abide by the legal requirements associated with these rights.

- Users may download and print one copy of any publication from the public portal for the purpose of private study or research.

- You may not further distribute the material or use it for any profit-making activity or commercial gain

- You may freely distribute the URL identifying the publication in the public portal

If you believe that this document breaches copyright please contact us providing details, and we will remove access to the work immediately and investigate your claim 


\title{
Geometric singular perturbation analysis of a dynamical target mediated drug disposition model
}

\author{
Kristian Uldall Kristiansen
}

Received: date / Accepted: date

\begin{abstract}
In this paper we present a mathematical analysis of a pharmacological ODE model for target mediated drug disposition (TMDD). It is known that solutions of this model undergo four qualitatively different phases. In this paper we provide a mathematical identification of these separate phases by viewing the TMDD model as a singular perturbed system. Our analysis is based on geometric singular perturbation theory and we believe that this approach systemizes - and sheds further light on - the scalings arguments used by previous authors. In particular, we present a novel description of the third phase through a distinguished solution of a nonlinear differential equation. We also describe the solution curve for large values of initial drug doses and recover, en route, a result by Aston et al, 2014, on rebounding using our alternative perturbation approach. Finally, from our main result we derive a new method for estimating the parameters of the system in the event that detailed data is available. Ideally our approach to the TMDD model should stimulate further research into applications of these methods to more complicated models in pharmacology.
\end{abstract}

Keywords Geometric singular perturbation theory · pharmacology · target mediated drug disposition

\section{Introduction}

In pharmacology, the main objective is to understand physiological processes and develop drugs that eliminate undesirable effects Peletier and Gabrielsson [2015]. Central to these objectives is data collection of the processes, e.g.

K. U. Kristiansen

Technical University of Denmark, Kgs. Lyngby, Denmark

Tel.: +45-45253063

E-mail: krkri@dtu.dk 
through measurements of blood pressure and heart rate. But for some processes, like neurological ones, data is obtained more indirectly in combination with mathematical modelling. For these reasons, mathematical analysis is now becoming increasingly relevant for drug development van der Graaf et al. [2016].

Pharmacological models are typically compartment-like models described by nonlinear ODEs. Here chemical reactions are described through rate constants. Frequently, such models exhibit, e.g. due to rate constants on separate orders of magnitude and/or as a consequence of the compartment structure, slow-fast phenomenon where variables alternate between slow and fast transitions. Mathematically, the slow-fast structure can be exploited using singular perturbation theory Jones [1995]; Kaper [1999]; Kuehn [2015]. In many situations (see e.g. Kosiuk and Szmolyan [2011, 2015, 2009]; Bossolini et al. [2017]) this theory, in combination with the blowup approach Krupa and Szmolyan [2001a], enables an accurate description of global phenomena (limit cycles, homoclinics, etc). This is typically not possible for general nonlinear differential equations without a slow-fast structure.

Target mediated drug disposition (TMDD) is the pharmacological phenomenon in which a drug binds with high affinity to its pharmacological target, such as a receptor, affecting its characteristics Levy [1994]; Dua et al. [2016]. In this paper we consider the following basic model

$$
\begin{aligned}
& \frac{d \tilde{x}}{d \tilde{t}}=-k_{\mathrm{on}} \tilde{x} \tilde{y}+k_{\mathrm{off}} \tilde{z}-k_{\mathrm{eL}} \tilde{x}, \\
& \frac{d \tilde{y}}{d \tilde{t}}=k_{\mathrm{in}}-k_{\mathrm{out}} \tilde{y}-k_{\mathrm{on}} \tilde{x} \tilde{y}+k_{\mathrm{off}} \tilde{z}, \\
& \frac{d \tilde{z}}{d \tilde{t}}=k_{\mathrm{on}} \tilde{x} \tilde{y}-\left(k_{\mathrm{off}}+k_{\mathrm{eRL}}\right) \tilde{z},
\end{aligned}
$$

introduced by Mager and Jusko [2001], where the drug (ligand) $\tilde{x}$ binds the target (receptor) $\tilde{y}$ to form the ligand-target complex $\tilde{z}$. The parameters $k_{i}, i=$ off, on, eL, eRL, in and out, are different rate constants associated with the process, see Peletier and Gabrielsson [2009, 2012] for more information. Since $\tilde{x}, \tilde{y}$ and $\tilde{z}$ measure concentration we restrict attention to the positive octant $\widetilde{\mathcal{O}}=\left\{(\tilde{x}, \tilde{y}, \tilde{z}) \in[0, \infty)^{3}\right\}$. Let

$$
\tilde{y}_{0}=\frac{k_{\text {in }}}{k_{\text {out }}} .
$$

Then the system (1) has a unique equilibrium

$$
\widetilde{\mathrm{Eq}}=\left(0, \tilde{y}_{0}, 0\right),
$$

inside $\widetilde{\mathcal{O}}$.

Following Peletier and Gabrielsson [2009, 2012], it is assumed that an initial drug dose is administered intravenously, while the system is in equilibrium. Therefore the relevant initial conditions for (1) are

$$
\widetilde{\mathrm{IC}}=\left(\tilde{x}_{0}, \tilde{y}_{0}, 0\right),
$$


Table 1 Values of the parameters from Peletier and Gabrielsson [2012].

\begin{tabular}{|c||c|c|c|c|c|c|c|}
\hline & $k_{\text {on }}$ & $k_{\text {off }}$ & $k_{\text {eL }}$ & $k_{\text {in }}$ & $k_{\text {out }}$ & $k_{\text {eRL }}$ & $\tilde{x}_{0}$ \\
\hline Value & 0.091 & 0.001 & 0.0015 & 0.11 & 0.0089 & 0.003 & 100 \\
\hline Unit & $(m g / L)^{-1} h^{-1}$ & $h^{-1}$ & $h^{-1}$ & $(m g / L) h^{-1}$ & $h^{-1}$ & $h^{-1}$ & $m g / L$ \\
\hline
\end{tabular}

where $\tilde{x}_{0}$ is the initial drug concentration. In this paper we will use $\tilde{x}_{0}=100$ in our computations. All values of the parameters from Peletier and Gabrielsson [2012] are displayed in Table 1.

Following Aston et al. [2014], the system (1) admits a unique, global forward solution inside the positive octant $\widetilde{\mathcal{O}}$. Moreover, the equilibrium $\widetilde{\mathrm{Eq}}$ is the global attractor. The evolution of the concentrations is shown in Fig. 1 (notice $\log -\log$ scale) for the parameter values in Table 1 , in particular $\tilde{x}_{0}=100$ and $\tilde{y}_{0}=12.3596$. Peletier and Gabrielsson [2012] then noted that the forward solution $(\tilde{x}(\tilde{t}), \tilde{y}(\tilde{t}), \tilde{z}(\tilde{t})), \tilde{t} \geq 0$, of (1) with $(\tilde{x}(0), \tilde{y}(0), \tilde{z}(0))=\widetilde{\mathrm{IC}}$ qualitatively undergoes four different phases $\widetilde{\mathbb{P}}^{1}, \ldots, \widetilde{\mathbb{P}}^{4}$. The phases are described as follows:

$\widetilde{\mathbb{P}}^{1}$ : In this initial phase, all variables exhibit significant variations over a relatively small time interval. The ligand $\tilde{x}$ binds the receptor $\tilde{y}$ and both variables decrease while $\tilde{z}$ is increasing. This happens in such a way that the total amount of ligand $\tilde{X}=\tilde{x}+\tilde{z}$ and the total amount of receptor $\tilde{Y}=\tilde{y}+\tilde{z}$ are both almost constant during this phase. The phase ends when the receptor $\tilde{y}$ is small enough.

$\widetilde{\mathbb{P}}^{2}$ : The subsequent phase is slower. Here $\tilde{y}$ increases gradually while the ligand $\tilde{x}$ is removed by the complex $\tilde{z}$ in the absence of receptor $\tilde{y}$. This phase ends when the concentration of the ligand is small enough.

$\widetilde{\mathbb{P}}^{3}:$ In this phase $\tilde{y}$ starts to increase more profoundly while $\tilde{x}$ continues to decrease. $\tilde{x}$ and $\tilde{y}$ appear to be fast variables during this phase whereas $\tilde{z}$ is almost constant. Phase $\widetilde{\mathbb{P}}^{3}$ is shorter than $\widetilde{\mathbb{P}}^{2}$ but significantly longer than $\widetilde{\mathbb{P}}^{1}$.

$\widetilde{\mathbb{P}}^{4}$ : In the final phase, the system contracts towards the unique equilibrium

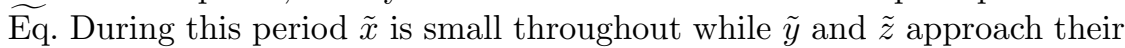
steady state values $\tilde{y}_{0}$ and 0 , respectively. In particular, $\tilde{z}$ is monotonically decreasing during this phase, whereas the receptor $\tilde{y}$, in general, see Aston et al. [2014], only increases monotonically during the first part. In the situation where $\tilde{y}$ is not monotone, it first exceeds $\tilde{y}_{0}$ before decreasing again. This is called rebounding. In Fig. 1, no rebounding occurs as $\tilde{y}$ is seen to increase monotonically towards it steady state value.

In e.g. [Peletier and Gabrielsson, 2013, Section 6], it is shown that the parameters in the model can be estimated by separation of the data into these separate phases. We will revisit this in Section 6.

Non-dimensionalisation. Following Peletier and Gabrielsson [2015], we consider the following non-dimensionalisation of the variables

$$
\tilde{x}=\tilde{y}_{0} x, \quad \tilde{y}=\tilde{y}_{0} y, \quad \tilde{z}=\tilde{y}_{0} z, \quad \tilde{t}=k_{\text {off }}^{-1} t .
$$




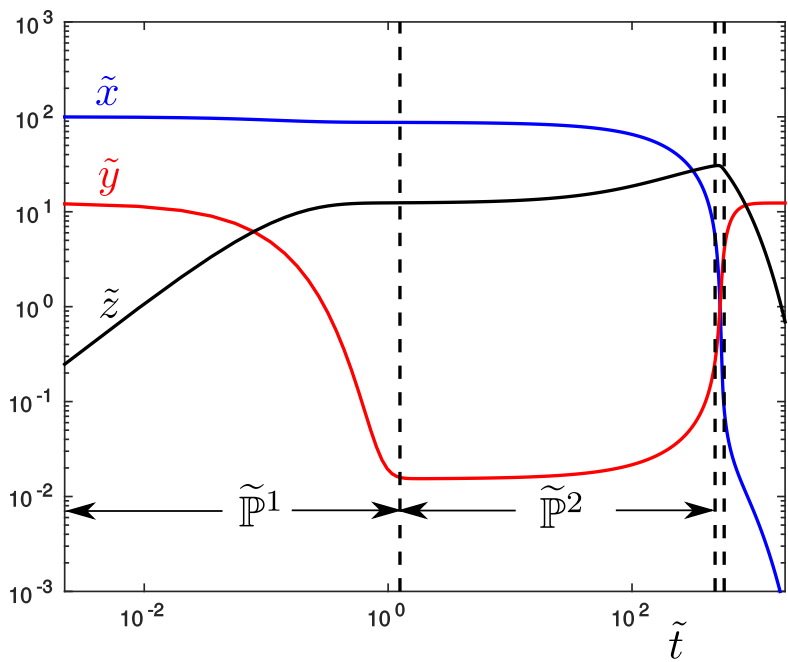

(a)

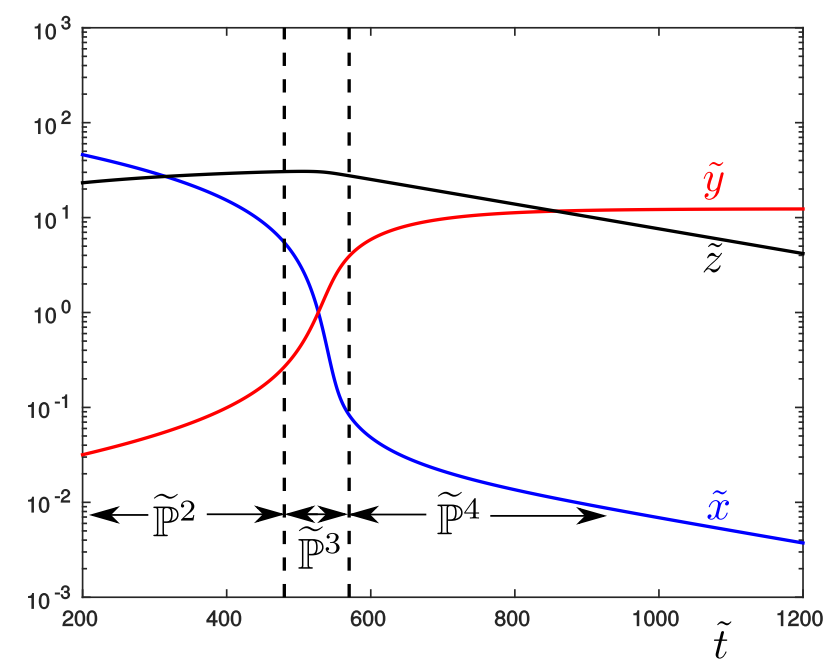

(b)

Fig. 1 The solution $(\tilde{x}(\tilde{t}), \tilde{y}(\tilde{t}), \tilde{z}(\tilde{t})), \tilde{t} \geq 0$, of (1) with $(\tilde{x}(0), \tilde{y}(0), \tilde{z}(0))=\widetilde{\mathrm{IC}}$ and the parameters in Table 1. The solution is asymptotic to Eq but undergoes four different phases $\widetilde{\mathbb{P}}^{1}, \ldots, \widetilde{\mathbb{P}}^{4}$, separated by vertical dashed lines in (a) and (b).

This gives the system

$$
\begin{aligned}
& \epsilon \dot{x}=-x y+\epsilon z-\epsilon \alpha x, \\
& \epsilon \dot{y}=-x y+\epsilon \beta(1-y)+\epsilon z, \\
& \epsilon \dot{z}=x y-\epsilon(1+\delta) z
\end{aligned}
$$


Table 2 Non-dimensionalised numbers. The values are computed from the dimensional numbers in Table 1. $\tilde{y}_{0}$ is defined in (2).

\begin{tabular}{|c||c|c|c|c|c|}
\hline & $\epsilon$ & $\alpha$ & $\beta$ & $\delta$ & $x_{0}$ \\
\hline Definition & $\frac{k_{\text {off }}}{k_{\text {on }} \tilde{y}_{0}}=\frac{k_{\text {off }} k_{\text {out }}}{k_{\text {on }} k_{\text {in }}}$ & $\frac{k_{\text {eL }}}{k_{\text {off }}}$ & $\frac{k_{\text {out }}}{k_{\text {off }}}$ & $\frac{k_{\text {eRL }}}{k_{\text {off }}}$ & $\frac{\tilde{x}_{0}}{\tilde{y}_{0}}=\frac{\tilde{x}_{0} k_{\text {out }}}{k_{\text {in }}}$ \\
\hline Value & $8.891 \times 10^{-4}$ & 1.500 & 8.900 & 3.000 & 8.091 \\
\hline
\end{tabular}

with new non-dimensional parameters $\epsilon, \alpha, \beta, \delta$ listed in Table 2. Here $\dot{()}=\frac{d}{d t}$ is differentiation with respect to the non-dimensional time $t$. Notice that $\epsilon=$ $8.891 \times 10^{-4}$ in Table 2 is small, and hence the velocities of $x, y$ and $z$ are all large in general. The non-dimensionalised initial conditions are

$$
\mathrm{IC} \equiv\left(x_{0}, 1,0\right)
$$

with

$$
x_{0}=\tilde{x}_{0} / \tilde{y}_{0}
$$

cf. (4).

Similarly, the unique non-negative equilibrium (3) in the original $(\tilde{x}, \tilde{y}, \tilde{z})$ variables becomes

$$
\mathrm{Eq} \equiv(0,1,0) .
$$

Remark 1 In Peletier and Gabrielsson [2012], $x$ is defined by $\tilde{x}=\tilde{x}_{0} x$. However, we prefer to use $x$ as in (5). We find this scaling more natural since then the differential equations are independent of $\tilde{x}_{0}$. Instead the initial condition on $x$ varies with $\tilde{x}_{0}$, which from a dynamical systems point of view seems more desirable.

For clarity we illustrate in Fig. 2 the non-dimensionalised version (using $(x, y, z))$ of the solution (using $(\tilde{x}, \tilde{y}, \tilde{z}))$ in Fig. 1. The phases in Fig. 2 are denoted by $\mathbb{P}^{1}, \ldots, \mathbb{P}^{4}$.

Known results. Peletier and Gabrielsson [2012, 2013] described the phases using scaling and asymptotic arguments, following Aston et al. [2011], and derived estimates of time and approximative equations for the evolution of the variables. In fact, the parameters $\alpha, \beta, \delta$ (called $\gamma$ ) and $\epsilon$ also appear in [Peletier and Gabrielsson, 2013, Eq. (5.3)] and it assumed, see [Peletier and Gabrielsson, 2013, (A) p. 604], that $\epsilon \ll 1$. But there are also other assumptions in these references, such as [Peletier and Gabrielsson, 2012, Assumption p. 439], which are derived from observations of numerical computations. They are not shown to be consequences of the initial assumptions. Since the approach is fairly adhoc, it is also unclear how robust their approach is for other, more complicated systems. 


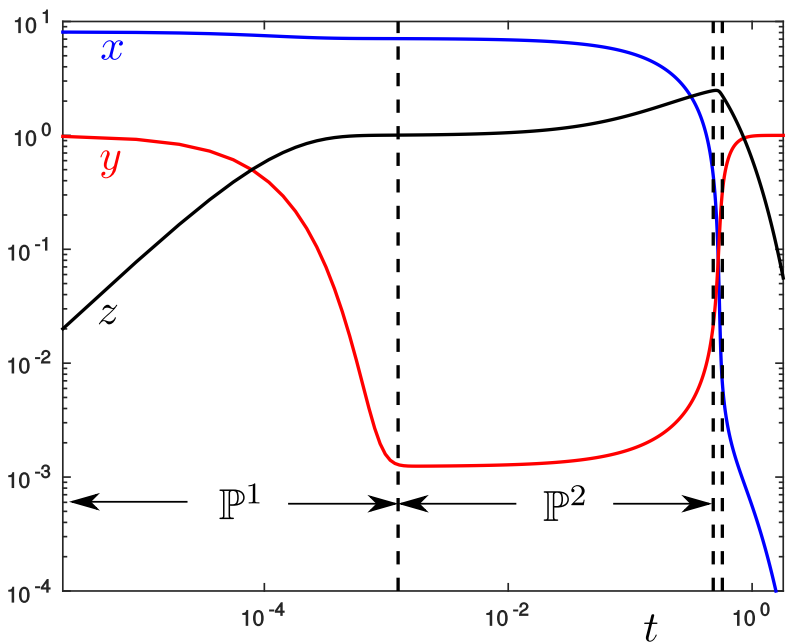

(a)

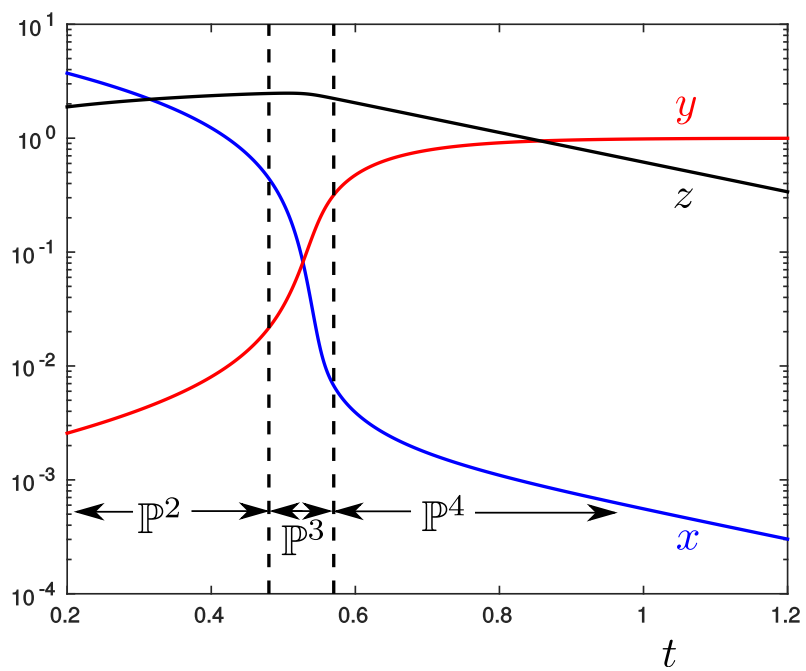

(b)

Fig. 2 The solution $(x(t), y(t), z(t)), t \geq 0$, of (6) with $(x(0), y(0), z(0))=\mathrm{IC}$ and the parameters in Table 2. The solution converges to Eq but undergoes four different phases $\mathbb{P}^{1}, \ldots, \mathbb{P}^{4}$, separated by vertical dashed lines.

Aim of the paper. The purpose of this paper is to provide a mathematical identification and quantitative description of each of the phases $\mathbb{P}^{1}, \ldots, \mathbb{P}^{4}$ by 
viewing (6) as a singular perturbation problem with

$$
\alpha, \beta, \delta, x_{0} \quad \text { all positive and } \quad 0<\epsilon \ll 1 \text {. }
$$

This will include a geometric analysis of the dynamics and a novel description of the third phase $\mathbb{P}^{3}$. In accordance with Table 2 , we will also suppose that

$$
x_{0}>1
$$

which in dimensional form becomes $\tilde{x}_{0}>\tilde{y}_{0}$.

Singular perturbation approach. Singular perturbations frequently occur in models of biological and bio-chemical processes, see Kosiuk and Szmolyan [2011, 2015]. Frequently such systems can be written in the standard slow-fast form

$$
\begin{aligned}
\epsilon \dot{u} & =f(u, v, \epsilon), \\
\dot{v} & =g(u, v, \epsilon),
\end{aligned}
$$

with $(u, v) \in \mathbb{R}^{m+n}$, smooth functions $f$ and $g$ and a small parameter $\epsilon>0$. Here $\left(\dot{)}=\frac{d}{d t}\right.$ and the time $t$ in (12) is said to be slow. The system (12) is therefore also called the slow system. Introducing the fast time scale $\tau=t / \epsilon$ gives the fast problem

$$
\begin{aligned}
& u^{\prime}=f(u, v, \epsilon), \\
& v^{\prime}=\epsilon g(u, v, \epsilon),
\end{aligned}
$$

where now ()$^{\prime}=\frac{d}{d \tau}$. It is possible to study systems like (12) or equivalently (13) for $0<\epsilon \ll 1$ using Fenichel's geometric theory of singular perturbations Fenichel [1971]. The point of departure for this theory is the two limiting systems: the reduced problem

$$
\begin{aligned}
& 0=f(u, v, 0), \\
& \dot{v}=g(u, v, 0),
\end{aligned}
$$

obtained from $(12)_{\epsilon=0}$, and the layer problem

$$
\begin{aligned}
& u^{\prime}=f(u, v, 0), \\
& v^{\prime}=0 .
\end{aligned}
$$

obtained from $(13)_{\epsilon=0}$. The equation $f(u, v, 0)=0$ defines a set of critical points $\mathcal{C}$ for $(15)$, called the critical manifold. On the other hand, the reduced problem is only defined on $\mathcal{C}$. A point $(u, v) \in \mathcal{C}$ is said to be normally hyperbolic if the Jacobian $\frac{\partial f}{\partial u}(u, v, 0)$ has no eigenvalues on the imaginary axis. Due to Fenichel, any compact normally hyperbolic submanifold $\mathcal{S} \subset \mathcal{C}$ perturbs smoothly to a locally invariant slow manifold $\mathcal{S}_{\epsilon}=\mathcal{S}+O(\epsilon)$ for $\epsilon$ sufficiently small. In particular, if $\mathcal{S}$ is a graph $u=h_{0}(v), v \in I$ then $\mathcal{S}_{\epsilon}$ is also a graph $u=h_{\epsilon}(u)=h_{0}(u)+O(\epsilon), v \in I$, smooth in $\epsilon$. Furthermore, the slow manifold 
$\mathcal{S}_{\epsilon}$ has stable and unstable manifolds, each of which has smooth invariant foliation by fibers, that are smooth $O(\epsilon)$-perturbations of the individual stable and unstable manifolds of points on $\mathcal{S}$ for (15). Consequently, orbits of (12) near $\mathcal{S}$ limit (in Hausdorff distance) as $\epsilon \rightarrow 0$ to singular orbits consisting of pieces of orbits of the reduced problem (14) and of the layer problem (15). See Jones [1995]; Kuehn [2015] for more details.

On the other hand, the slow system (6), or equivalently the corresponding fast system

$$
\begin{aligned}
& x^{\prime}=-x y+\epsilon z-\epsilon \alpha x, \\
& y^{\prime}=-x y+\epsilon \beta(1-y)+\epsilon z, \\
& z^{\prime}=x y-\epsilon(1+\delta) z,
\end{aligned}
$$

is an example of a system in nonstandard slow-fast form:

$$
w^{\prime}=F(w, \epsilon), \quad w \in \mathbb{R}^{m+n} .
$$

see also Kosiuk and Szmolyan [2015]; Kosiuk [2012]; Goeke et al. [2015]. In (16), ()$^{\prime}=\frac{d}{d \tau}$ and $\tau=\epsilon^{-1} t$ is the fast time. Fenichel's theory applies to (17) as follows: Suppose $F(w, 0)=0$ (or a subset hereof) is a compact $n$-dimensional critical manifold $\mathcal{S}$ satisfying the normal hyperbolicity assumption: For any point $w \in \mathcal{S}$, the Jacobian $\frac{\partial F}{\partial w}(w, 0)$ has only $n$ eigenvalues with zero real part, then $\mathcal{S}$ perturb smoothly to a locally invariant slow manifold $\mathcal{S}_{\epsilon}=\mathcal{S}+O(\epsilon)$, for $\epsilon$ sufficiently small, again with stable and unstable manifolds. The stable and unstable manifolds have smooth invariant foliation by fibers, that are smooth $O(\epsilon)$-perturbations of the individual stable and unstable, respectively, manifolds of points on $\mathcal{S}$ for the following layer problem $(17)_{\epsilon=0}$ :

$$
w^{\prime}=F(w, 0) .
$$

Furthermore, suppose for simplicity that

$$
w=(u, v) \in \mathbb{R}^{m+n}, \quad F(w, \epsilon)=\left(F_{u}(u, v, \epsilon), F_{v}(u, v, \epsilon)\right)^{T},
$$

and that $\mathcal{S}$ can be written as a graph $u=h_{0}(v)$. Then $\mathcal{S}$ is normally hyperbolic if all the eigenvalues of the matrix

$$
P(v)=\left(\frac{\partial h_{0}}{\partial v}(v) \frac{\partial F_{v}}{\partial u}\left(h_{0}(v), v, 0\right)-\frac{\partial F_{u}}{\partial u}\left(h_{0}(v), v, 0\right)\right) \in \mathbb{R}^{m \times m},
$$

have non-zero real part. In particular, $P$ is then a regular matrix for each $v$. Furthermore, the slow flow on $\mathcal{S}_{\epsilon}$ is on the slow time-scale smoothly $O(\epsilon)$-close to the reduced system:

$$
\dot{w}=\frac{\partial F}{\partial u}(w, 0) h_{1}(v)+\frac{\partial F}{\partial \epsilon}(w, 0),
$$

on $w \in \mathcal{S} \subset \mathbb{R}^{n+m}$, where

$$
h_{1}(v)=P(v)^{-1}\left(\frac{\partial F_{u}}{\partial \epsilon}\left(h_{0}(v), v, 0\right)-\frac{\partial h_{0}}{\partial v}(v) \frac{\partial F_{v}}{\partial \epsilon}\left(h_{0}(v), v, 0\right)\right) .
$$


See e.g. Goeke et al. [2015]. (In fact, $h_{1}$ is such that $\mathcal{S}_{\epsilon}=\left\{(u, v) \mid u=h_{0}(v)+\right.$ $\left.\epsilon h_{1}(v)+O\left(\epsilon^{2}\right)\right\}$.)

Remark 2 Locally, it is always possible to straighten out orbits of the layer problem and transform a slow-fast system in nonstandard form into a slow-fast system in standard form. For (16), we notice that

$$
\begin{aligned}
& (x+z)^{\prime}=-\epsilon(\alpha x+\delta z), \\
& (y+z)^{\prime}=\epsilon(\beta(1-y)-\delta z) .
\end{aligned}
$$

Therefore a (global) transformation to standard form is obtained by introducing

$$
\begin{aligned}
& X=x+z, \\
& Y=y+z
\end{aligned}
$$

as the total ligand and total receptor, and considering $(X, Y, z)$ as new variables. Then $X$ and $Y$ are slow variables while $z$ is fast. This is the approach taken in Peletier and Gabrielsson [2012, 2013] to describe the second phase. However, as the present paper exemplifies, there is no gain in going to standard slow-fast form. The analysis and the geometry is not simpler. On the contrary, working with an applied problem, there is a general advantage in using the scientifically meaningful variables. In our case, this also eases the comparison with the numerical computations in Fig. 1. We therefore proceed working with the system in nonstandard form (16).

Outline. In the following Section 2 we will begin our singular perturbation analysis of (1). For this we consider the scaled variables $(x, y, z)$ and restrict attention to the forward invariant positive octant $\mathcal{O}=\left\{(x, y, z) \in[0, \infty)^{3}\right\}$. In Section 3 we present our main result, Theorem 1, which describes the limit of the forward orbit of IC for $\epsilon \rightarrow 0$ with fixed $x_{0}>1$, as the union of orbits of the layer- and reduced problem. In Theorem 2 we describe $x_{0} \rightarrow \infty$, by showing that the convergence of the forward orbit to the limiting orbit in Theorem 1 is uniform in $x_{0}>1$ within any fixed compact set. To the author's knowledge, this result has not been reported in the literature at any level of formality. We prove Theorem 1 in Section 4 and Theorem 2 in Appendix B. We illustrate our results using numerical computations in Section 5. In Section 6 we conclude the manuscript with a discussion section, including a discussion of the role of the parameters and a description of a new procedure to estimate these.

The proof of Theorem 1 shows that the third phase $\mathbb{P}^{3}$ is described by the regime $x=O(\sqrt{\epsilon}), y=O(\sqrt{\epsilon})$, which we capture by the scaling

$$
x=\sqrt{\epsilon} x_{2}, \quad y=\sqrt{\epsilon} y_{2},
$$

see also Section 4.1. This scaling gives rise to an inner solution which approximates the third phase $\mathbb{P}^{3}$, see illustration in Fig. 9 in Section 5. In terms of 
the dimensional variables, this scaling becomes

$$
\begin{aligned}
& \tilde{x}=\tilde{y}_{0} \sqrt{\epsilon} x_{2}=\sqrt{\frac{\tilde{y}_{0} k_{\mathrm{off}}}{k_{\mathrm{on}}}} x_{2}=\sqrt{\frac{k_{\mathrm{in}} k_{\mathrm{off}}}{k_{\mathrm{out}} k_{\mathrm{on}}}} x_{2}, \\
& \tilde{y}=\tilde{y}_{0} \sqrt{\epsilon} y_{2}=\sqrt{\frac{\tilde{y}_{0} k_{\mathrm{off}}}{k_{\mathrm{on}}}} y_{2}=\sqrt{\frac{k_{\mathrm{in}} k_{\mathrm{off}}}{k_{\mathrm{out}} k_{\mathrm{on}}}} y_{2} .
\end{aligned}
$$

The scaling in (20) is therefore different from the one used in [Peletier and Gabrielsson, 2013, Eq. (5.17)] and [Peletier and Gabrielsson, 2012, Eq. (32)] and our description of the third phase therefore differs from theirs, see also Remark 5. Notation: We will enumerate certain geometric objects that appear in our analysis using superscripts. These superscripts are enumerated $1, \ldots, 4$ according to the phase $\mathbb{P}^{1}, \ldots, \mathbb{P}^{4}$ they describe or relate to.

\section{Geometric singular perturbation analysis of (16)}

Our singular perturbation analysis of (1) initially rests upon the layer problem and the reduced problem. We consider these systems successively in the following.

\subsection{Layer problem}

Setting $\epsilon=0$ in (16) gives the layer problem:

$$
\begin{aligned}
& x^{\prime}=-x y, \\
& y^{\prime}=-x y, \\
& z^{\prime}=x y .
\end{aligned}
$$

We have the following (see Fig. 3)

Lemma 1 The sets

$$
\begin{aligned}
& \mathcal{S}^{2}=\{(x, y, z) \in \mathcal{O} \mid x>0, y=0\}, \\
& \mathcal{S}^{4}=\{(x, y, z) \in \mathcal{O} \mid x=0, y>0\}, \\
& \mathcal{L}^{3}=\{(x, y, z) \in \mathcal{O} \mid x=y=0\},
\end{aligned}
$$

are sets of critical points for (6). $\mathcal{S}^{2}$ and $\mathcal{S}^{4}$ are both normally hyperbolic and attracting whereas $\mathcal{L}^{3}$ is a line of fully nonhyperbolic points.

Consider the plane

$$
\mathcal{F}=\{(x, y, z) \in \mathcal{O} \mid y=x, x>0\}
$$

and let

$$
\begin{aligned}
& \mathcal{F}^{+}=\{(x, y, z) \in \mathcal{O} \mid y>x, x>0\}, \\
& \mathcal{F}^{-}=\{(x, y, z) \in \mathcal{O} \mid y<x, x>0\},
\end{aligned}
$$

be the region above and below $\mathcal{F}$, respectively. Then: 
- All points within $\mathcal{F}^{-}$are forward asymptotic under the flow of (21) to a point on $\mathcal{S}^{2}$

- All points within $\mathcal{F}$ are forward asymptotic under the flow of (21) to a point on $\mathcal{L}^{3}$.

- All points within $\mathcal{F}^{+}$are forward asymptotic under the flow of (21) to a point on $\mathcal{S}^{4}$.

Proof The linearization of any point in $\mathcal{S}^{4}$ gives two zero eigenvalues and one negative $-y$. Similarly, the linearization about any point in $\mathcal{S}^{2}$ gives two zero eigenvalues and one negative $-x$. On the other hand, along $\mathcal{L}^{3}$ the linearization has only zero eigenvalues.

For the statement about $\mathcal{F}, \mathcal{F}^{ \pm}$, we notice that away from $\mathcal{S}^{2}, \mathcal{L}^{3}$ and $\mathcal{S}^{4}$ we can divide the right hand side by $x y>0$. Let therefore $\tau_{1}$ be defined as follows

$$
\frac{d \tau_{1}}{d \tau}=x(\tau) y(\tau)>0
$$

Then, by the chain rule, the division of the right hand side by $x y>0$, corresponds to a re-parametrization of time by $\tau_{1}=\tau_{1}(\tau)$ such that

$$
\begin{aligned}
& \frac{d x}{d \tau_{1}}=-1, \\
& \frac{d y}{d \tau_{1}}=-1 \\
& \frac{d z}{d \tau_{1}}=1
\end{aligned}
$$

Orbits of (21) are therefore of the form

$$
(x, y, z)=\left(c_{1}, c_{2}, c_{3}\right)+(-1,-1,1) \tau_{1},
$$

$\tau_{1} \in \mathcal{I}, \mathcal{I}=(a, b)$ an appropriate interval. These curves produce the critical fibers of points on $\mathcal{S}^{2}, \mathcal{S}^{4}$ and $\mathcal{L}^{3}$. Letting $\left(c_{1}, c_{2}, c_{3}\right) \in \mathcal{L}^{3}$, so that $c_{1}=c_{2}=0$, produces $\mathcal{F}$. The result then follows.

\subsection{Reduced problem}

To compute the reduced problem on $\mathcal{S}^{2}$ and $\mathcal{S}^{4}$, we use (18). This gives $h_{1}(x, z)=x^{-1}(z+\beta)$ with $u=y, v=(x, z), P(v)=x$ and $h_{1}(y, z)=y^{-1} z$ with $u=x, v=(y, z), P(v)=y$, respectively, in (18). Therefore we obtain the following two linear systems:

$$
\begin{aligned}
& \dot{x}=-\beta-\alpha x, \\
& y=0, \\
& \dot{z}=\beta-\delta z,
\end{aligned}
$$


and

$$
\begin{aligned}
& x=0, \\
& \dot{y}=\beta(1-y), \\
& \dot{z}=-\delta z,
\end{aligned}
$$

on $\mathcal{S}^{2}$ and $\mathcal{S}^{4}$, respectively. Any point $(x, y, z)=\left(0, c_{2}, c_{3}\right)$ within $\mathcal{S}^{4}$ is therefore, under the forward flow of the reduced problem (24), given by

$$
y(t)=1+\left(c_{2}-1\right) e^{-\beta t}, \quad z(t)=c_{3} e^{-\delta t},
$$

asymptotic to the stable node at $(x, y, z)=\mathrm{Eq}$, recall (9). Within $\{z \geq 0, y<$ $1, x=0\}, y$ is monotonically increasing towards $y=1$ while $z$ is monotonically decreasing towards $z=0$. Similarly, any point within $\mathcal{S}^{2}$ reaches, under the forward flow of the reduced problem (23), its boundary along $\mathcal{L}^{3}$ in finite time. In details, if $x(0)=c_{1}, z(0)=c_{3}$, then

$$
x(t)=-\alpha^{-1} \beta+\left(c_{1}+\alpha^{-1} \beta\right) e^{-\alpha t}, \quad z(t)=\delta^{-1} \beta+\left(c_{3}-\delta^{-1} \beta\right) e^{-\delta t},
$$

for $t \in\left[0, T\left(c_{1}\right)\right)$, for

$$
T\left(c_{1}\right)=\alpha^{-1} \ln \left(\frac{c_{1}+\alpha^{-1} \beta}{\alpha^{-1} \beta}\right)
$$

where $x(t) \rightarrow 0^{+}$for $t \rightarrow T\left(c_{1}\right)$. Here $z$ is increasing below $z=\delta^{-1} \beta$ which is an invariant for the linear system (23) (corresponding to $c_{3}=\delta^{-1} \beta$ in (25)). We sketch the phase portraits of (23) and (24) within $\mathcal{S}^{2}$ and $\mathcal{S}^{4}$ in Fig. 3.

\subsection{Slow manifolds}

By Fenichel's theory Fenichel [1971], compact sub-manifolds of the normally hyperbolic critical manifolds $\mathcal{S}^{2}$ and $\mathcal{S}^{4}$ perturb to locally invariant slow manifolds $\mathcal{S}_{\epsilon}^{2}$ and $\mathcal{S}_{\epsilon}^{4}$, respectively. In details, we have

Lemma 2 Let $\mathcal{V}(\mathcal{W})$ be a large, closed rectangle in the positive quadrant of the $(x, z)$-plane $((y, z)$-plane $)$. Then there exists an $\epsilon_{0}=\epsilon_{0}(\mathcal{V}, \mathcal{W})>0$ and smooth functions

$$
G: \mathcal{V} \times\left[0, \epsilon_{0}\right] \rightarrow \mathbb{R}
$$

and

$$
H: \mathcal{W} \times\left[0, \epsilon_{0}\right] \rightarrow \mathbb{R}
$$

satisfying

$$
G(x, z, 0)=x^{-1}(z+\beta), \quad H(y, z, 0)=y^{-1},
$$

and

$$
\begin{aligned}
& \frac{\partial G}{\partial \epsilon}(x, z, 0)=-x^{-2}\left(\beta(z+\beta) x^{-1}+(\alpha+\beta)(z+\beta)+\beta-\delta z\right), \\
& \frac{\partial H}{\partial \epsilon}(y, z, 0)=y^{-2}\left(\delta-\alpha-\beta\left(1-y^{-1}\right)\right),
\end{aligned}
$$


such that the graphs

$$
\begin{aligned}
& \mathcal{S}_{\epsilon}^{2}=\{(x, y, z) \in \mathcal{O} \mid y=\epsilon G(x, z, \epsilon), \quad(x, z) \in \mathcal{V}\}, \\
& \mathcal{S}_{\epsilon}^{4}=\{(x, y, z) \in \mathcal{O} \mid x=\epsilon z H(y, z, \epsilon), \quad(y, z) \in \mathcal{W}\},
\end{aligned}
$$

are locally invariant attracting slow manifolds of (6) for $\epsilon \leq \epsilon_{0}$.

Also, $\mathcal{S}_{\epsilon}^{2}$ and $\mathcal{S}_{\epsilon}^{4}$ have $3 D$ stable manifolds with invariant foliations by stable fibers. These fibers are smoothly $O(\epsilon)$-close to the critical fibers that are orbits of the layer problem (21) asymptotic to points on $\mathcal{S}^{2}$ and $\mathcal{S}^{4}$, respectively. The contraction towards $\mathcal{S}_{\epsilon}^{2}$ and $\mathcal{S}_{\epsilon}^{4}$ along the fibers is $O\left(e^{-c t / \epsilon}\right), c=c(\mathcal{V}, \mathcal{W})>0$, on the slow time scale.

Proof Follows from Fenichel's theory and simple calculations. In particular, the form of $\mathcal{S}_{\epsilon}^{4}$ follows from the fact that $x=z=0$ is invariant for all $\epsilon>0$.

The slow flow on $\mathcal{S}_{\epsilon}^{2}$ and $\mathcal{S}_{\epsilon}^{4}$ is found by substituting the expressions (29) and (30) into (6). To order $O\left(\epsilon^{2}\right)$, we obtain

$$
\begin{aligned}
& \dot{x}=-\beta-\alpha x+\epsilon x^{-1}\left(\beta(z+\beta) x^{-1}+(\alpha+\beta)(z+\beta)+\beta-\delta z+\mathcal{O}(\epsilon)\right), \\
& \dot{z}=\beta-\delta z-\epsilon x^{-1}\left(\beta(z+\beta) x^{-1}+(\alpha+\beta)(z+\beta)+\beta-\delta z+\mathcal{O}(\epsilon)\right),
\end{aligned}
$$

and

$$
\begin{aligned}
& \dot{y}=\beta(1-y)-\epsilon z y^{-1}\left(\delta-\alpha-\beta\left(1-y^{-1}\right)+\mathcal{O}(\epsilon)\right), \\
& \dot{z}=-\delta z+\epsilon z y^{-1}\left(\delta-\alpha-\beta\left(1-y^{-1}\right)+\mathcal{O}(\epsilon)\right),
\end{aligned}
$$

respectively. Notice that we obtain (23) and (24) for $\epsilon=0$ as desired.

\section{Main result}

We now define a singular or candidate forward orbit of the initial condition IC:

$$
\Gamma_{0}=\Gamma^{1} \cup \Gamma^{2} \cup \gamma^{3} \cup \Gamma^{4}
$$

see Fig. 3, as the following concatenation of orbits of the layer problem and the reduced problem on $\mathcal{S}^{2}$ and $\mathcal{S}^{4}$ :

$-\Gamma^{1}$ is the forward orbit of IC under the flow of the layer problem (21). It is asymptotic to the base point

$$
\gamma^{2}=\left(x^{(2)}, 0,1\right) \in \mathcal{S}^{2},
$$

where

$$
x^{(2)}=x_{0}-1 .
$$

Notice that $x^{(2)}>0$ by assumption (11). In details,

$$
\Gamma^{1}=\left\{(x, y, z) \in \mathcal{O} \mid x=x_{0}-s, y=1-s, z=s, s \in[0,1)\right\},
$$

using (22) with $\left(c_{1}, c_{2}, c_{3}\right)=\left(x_{0}, 1,0\right)$. 
- $\Gamma^{2}$ is the forward orbit of $\gamma^{2} \in \mathcal{S}^{2}$ under the flow of the reduced problem $(23)$ on $\mathcal{S}^{2}$. It reaches

$$
\gamma^{3}=\left(0,0, z^{(3)}\right) \in \mathcal{L}^{3}
$$

where

$$
z^{(3)}=\delta^{-1} \beta+\left(1-\delta^{-1} \beta\right) e^{-\delta T\left(x^{(2)}\right)},
$$

in finite slow time

$$
T\left(x^{(2)}\right)=\alpha^{-1} \ln \left(\frac{x^{(2)}+\alpha^{-1} \beta}{\alpha^{-1} \beta}\right),
$$

recall (26). In details:

$$
\begin{aligned}
\Gamma^{2}=\left\{(x, y, z) \in \mathcal{S}^{2} \mid x(t)\right. & =-\alpha^{-1} \beta+\left(x^{(2)}+\alpha^{-1} \beta\right) e^{-\alpha t} \\
z(t) & \left.\left.=\delta^{-1} \beta+\left(1-\delta^{-1} \beta\right) e^{-\delta t}, t \in\left[0, T\left(x^{(2)}\right)\right)\right)\right\} .
\end{aligned}
$$

$-\Gamma^{4}$ is the orbit segment of $(24)$ on $\mathcal{S}^{4}$ that leaves $\mathcal{S}^{4}$ in finite backward time at $\gamma^{3}$ and is forward asymptotic to Eq. In details:

$$
\begin{aligned}
\Gamma^{4}=\left\{(x, y, z) \in \mathcal{S}^{4} \mid y(t)\right. & =1-e^{-\beta\left(t-T\left(x^{(2)}\right)\right)} \\
z(t) & \left.=z^{(3)} e^{-\delta\left(t-T\left(x^{(2)}\right)\right)}, t>T\left(x^{(2)}\right)\right\} .
\end{aligned}
$$

Then we have the following:

Theorem 1 Let $\Gamma_{\epsilon}$ be the forward orbit of IC under the flow of (16) and suppose that $\alpha, \beta, \delta$ are all positive and that (11) holds. Then there exists an $\epsilon_{0}>0$ such that for all $\epsilon \in\left(0, \epsilon_{0}\right]$ the following holds:

(A) $\Gamma_{\epsilon}$ is $O(\sqrt{\epsilon})$-close to $\Gamma_{0}$ in Hausdorff distance: $\Gamma_{\epsilon} \rightarrow \Gamma_{0}$ as $\epsilon \rightarrow 0^{+}$.

(B) $\Gamma_{\epsilon}$ is forward asymptotic to the equilibrium $\mathrm{Eq}(9)$.

Remark 3 Statement (B) follows from (A) since (a) $\Gamma^{4}$ is asymptotic to Eq and (b) Eq is a stable node of the reduced problem (31) for any $0<\epsilon \ll 1$. But (B) is not new. In fact, (B) is true for any $\epsilon>0$, see Aston et al. [2014] where separate methods were used. But, although our approach only works for $\epsilon \ll 1$, we believe that it is a more direct approach, that offers more explicit information about the dynamics, and which, at least potentially, may be more useful for more complicated systems of this kind. 


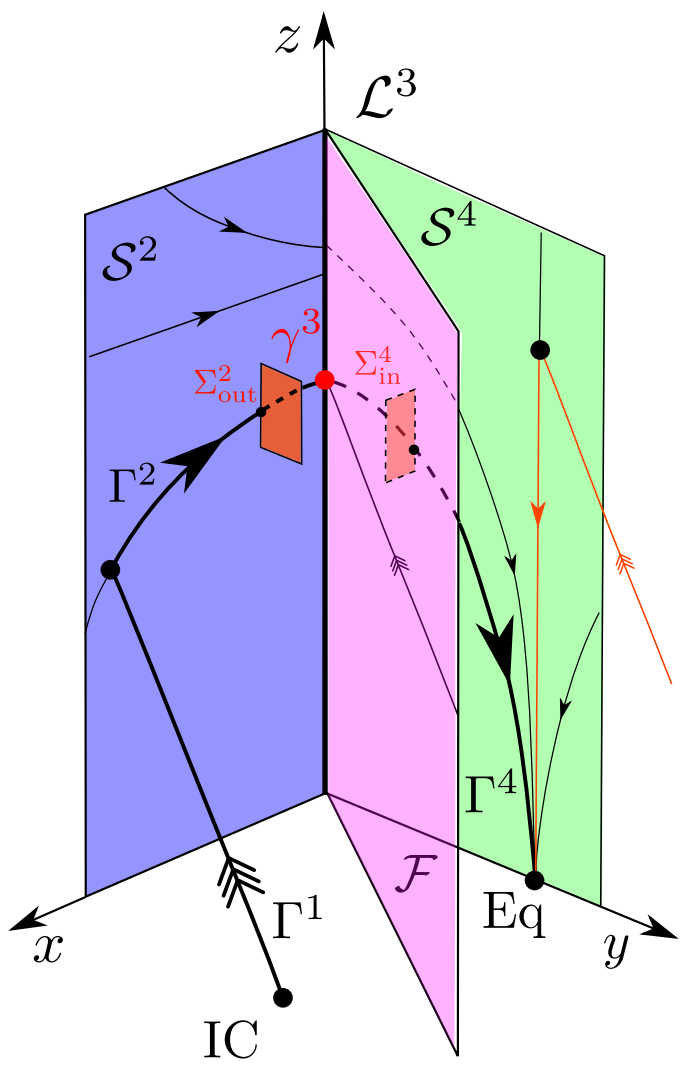

Fig. 3 Singular forward orbit $\Gamma_{0} . \Gamma^{1}$ is the forward orbit of IC under the flow of the layer problem. $\Gamma^{2}$ is a forward orbit of the base point of $\Gamma^{1}$ on $\mathcal{S}^{2}$ by the flow of the reduced problem. $\gamma^{3}$ is a point on $\mathcal{L}^{3}$. Finally $\Gamma^{4}$ is the orbit segment of the reduced problem on $\mathcal{S}^{4}$ that leaves $\mathcal{S}^{4}$ at $\gamma^{3}$ in finite backwards time. The sections $\Sigma_{\text {out }}^{2}$ and $\Sigma_{\text {in }}^{4}$ are used in the proof. The orange curve is an example of a singular orbit for $x_{0}<1$. Here the forward flow connects directly to a point on $\mathcal{S}^{4}$ through a stable critical fiber.

Remark 4 Theorem 1 describes the closeness of $\Gamma_{\epsilon}$ to $\Gamma_{0}$. The closeness of $\Gamma_{\epsilon}$ to the individual segments is described more carefully in Section 4.5. But essentially, the result says, loosely speaking, that outside a neighborhood of $\gamma^{3}, \Gamma_{\epsilon}$ is $O(\epsilon)$-close to $\Gamma^{1} \cup \Gamma^{2}$, but after passage near $\mathcal{L}^{3}$, it is only $O(\sqrt{\epsilon})$ close to $\Gamma^{4}$. To the author's knowledge, this order of accuracy has not been described previously in the literature. In particular, it does not follow from the analysis in Peletier and Gabrielsson [2012, 2013].

In Peletier and Gabrielsson [2012]; Patsatzis et al. [2016] larger values of $\tilde{x}_{0}$ are considered. This corresponds to larger values of $x_{0}$ by (8). Fenichel's theory only applies to compact critical manifolds. But when $x_{0}$ increases then the base point $\gamma^{2}$ moves further away from $\mathcal{L}^{3}$. Hence this may potentially 
require smaller $\epsilon$-values for (a) Fenichel's theory to apply near $\gamma^{2}$ and for (b) $\Gamma_{\epsilon}$ to be approximated by the singular orbit $\Gamma_{0}$. However, this is not the case for (1) in the sense of the following theorem.

Theorem 2 In the following, denote $\Gamma_{\epsilon}$ and $\Gamma_{0}$ for $x(0)=x_{0}$ by $\Gamma_{\epsilon}\left(x_{0}\right)$ and $\Gamma_{0}\left(x_{0}\right)$, respectively. Fix any bounded box $\mathcal{B}=\left[0, b_{1}\right] \times\left[0, b_{2}\right] \times\left[0, b_{3}\right]$ of xyzspace with $b_{i}>0, i=1,2,3$. Then the convergence $\Gamma_{\epsilon}\left(x_{0}\right) \cap \mathcal{B} \rightarrow \Gamma_{0}\left(x_{0}\right) \cap \mathcal{B}$ for $\epsilon \rightarrow 0$ is uniform with respect to $x_{0}>1$. In particular, there exists an $\epsilon_{0}$ and a sufficiently large $K>0$ such that

$$
\operatorname{dist}_{\text {Hausdorff }}\left(\Gamma_{\epsilon}\left(x_{0}\right) \cap \mathcal{B}, \Gamma_{0}\left(x_{0}\right) \cap \mathcal{B}\right) \leq K \sqrt{\epsilon}
$$

for all $\left(\epsilon, x_{0}\right) \in\left(0, \epsilon_{0}\right] \times(1, \infty)$.

Proof The proof builds upon the proof of Theorem 1. For further details, see Appendix B.

\section{Proof of Theorem 1}

By Fenichel's theory we can guide $\Gamma_{\epsilon}$ along $\Gamma^{1}$ and $\Gamma^{2}$ while $(x(t), z(t)) \in \mathcal{V}$ (recall Lemma 2) using the slow manifold $\mathcal{S}_{\epsilon}^{2}$ and its stable perturbed fibers. In particular, let $\xi>0$ be small, let $\mathcal{R}^{2}$ be a suitable rectangle in the $y z$-plane and denote the intersection of $\Gamma^{2}$ with the section $\Sigma_{\text {out }}^{2}=\left\{x=\xi,(y, z) \in \mathcal{R}^{2}\right\}$ (transverse to $\Gamma^{2}$ ) by $q^{1}$. Then $\Gamma_{\epsilon}$ intersects $\Sigma_{\text {out }}^{2}$ near $q^{1}$ in a point $q_{\epsilon}^{1}$ which is $O(\epsilon)$-close to $q^{1}$. Here $q_{\epsilon}^{1}$ is exponentially close to $\mathcal{S}_{\epsilon}^{2}$. Similarly, using the slow manifold $\mathcal{S}_{\epsilon}^{4}$, we can also guide initial conditions within the section $\Sigma_{\text {in }}^{4}=\{y=$ $\left.\xi,(x, z) \in \mathcal{R}^{4}\right\}$, sufficiently close to $\Gamma^{4}$, towards the equilibrium Eq. $\mathcal{R}^{4}$ is now a suitable rectangle in the $x z$-plane. But Fenichel's theory breaks down near $\mathcal{L}^{3}$ since this set is not normally hyperbolic, recall Lemma 1 , and as a result there is a gap between $\Sigma_{\text {out }}^{2}$ and $\Sigma_{\text {in }}^{4}$ that we need to cover to complete the proof of the theorem. This gap will relates to the third phase $\mathbb{P}^{3}$. We illustrate $\Sigma_{\text {out }}^{2}$ and $\Sigma_{\text {in }}^{4}$ in Fig. 3.

4.1 Dynamics near $\mathcal{L}^{3}$ : Regime $x, y=O(\sqrt{\epsilon})$

Consider the following scaling

$$
x=r_{2} x_{2}, \quad y=r_{2} y_{2}, \quad \epsilon=r_{2}^{2},
$$

zooming in on $\mathcal{L}^{3}$. Notice that (36) implies (19) since $r_{2}=\sqrt{\epsilon}$. Inserting $(36)$ into (16) gives

$$
\begin{aligned}
x_{2}^{\prime} & =r_{2}\left(-x_{2} y_{2}+z-r_{2} \alpha x_{2}\right), \\
y_{2}^{\prime} & =r_{2}\left(\beta\left(1-r_{2} y_{2}\right)-x_{2} y_{2}+z\right), \\
z^{\prime} & =r_{2}^{2}\left(x_{2} y_{2}-(1+\delta) z\right) . \\
r_{2}^{\prime} & =0 .
\end{aligned}
$$


Notice that $r_{2}$ is a common factor on the right hand side. Introducing a new time $\tau_{2}$ by

$$
\tau_{2}=r_{2} \tau
$$

gives

$$
\begin{aligned}
\dot{x}_{2} & =-x_{2} y_{2}+z-r_{2} \alpha x_{2}, \\
\dot{y}_{2} & =\beta\left(1-r_{2} y_{2}\right)-x_{2} y_{2}+z, \\
\dot{z} & =r_{2}\left(x_{2} y_{2}-(1+\delta) z\right) .
\end{aligned}
$$

where $\dot{(})=\frac{d}{d \tau_{2}}$, obtained from (37) by dividing the right hand side by $r_{2}$. Now, since $r_{2}=\sqrt{\epsilon} \ll 1$ the scaled variables $x_{2}$ and $y_{2}$ are both fast on the $\tau_{2}$ time scale while $z$ is slow. Setting $r_{2}=0$ gives a new layer problem

$$
\begin{aligned}
\dot{x}_{2} & =-x_{2} y_{2}+z, \\
\dot{y}_{2} & =\beta-x_{2} y_{2}+z, \\
\dot{z} & =0,
\end{aligned}
$$

where $z \in \mathbb{R}$ is now a parameter. There are no equilibria solutions of this system. Instead we have the following.

Lemma 3 Let $z \in[0, \infty)$. Then any solution $\left(x_{2}\left(\tau_{2}\right), y_{2}\left(\tau_{2}\right), z\right)$ of $(39)$, with initial conditions $\left(x_{2}(0), y_{2}(0)\right)$ in the first quadrant of the $\left(x_{2}, y_{2}\right)$-plane, is asymptotic to

$$
x_{2}=y_{2}^{-1} z,
$$

as $\tau_{2} \rightarrow \infty$. In particular, for each $z \in \mathbb{R}$ there exists a unique solution curve $\Gamma_{2}(z)$ of (39) with the following asymptotics

$$
y_{2}=x_{2}^{-1}(z+\beta),
$$

for $\tau_{2} \rightarrow-\infty$. Along $\Gamma_{2}(z), x_{2}$ decreases monotonically while $y_{2}$ increases monotonically.

Proof Follows from (a) a simple phase plane analysis in the $x_{2} y_{2}$-plane using nullclines and the positive invariance of the first quadrant in the $\left(x_{2}, y_{2}\right)$-plane, see Fig. 4, and (b) the existence of locally invariant manifolds at $x_{2} \gg 1$ and $y_{2} \gg 1$. The manifold with $x_{2} \gg 1$ is unique whereas the one with $y_{2} \gg 1$ is not. (b) follows from a Poincaré-like compactification of the $\left(x_{2}, y_{2}\right)$-plane and center manifold theory. Further details are delayed to Appendix A.

Now, on a purely formal level we note the following: Consider the following approximation of the slow manifolds $\mathcal{S}_{\epsilon}^{2}$ and $\mathcal{S}_{\epsilon}^{4}$ :

$$
\begin{aligned}
& y \approx \epsilon x^{-1}(z+\beta), \\
& x \approx \epsilon y^{-1} z,
\end{aligned}
$$




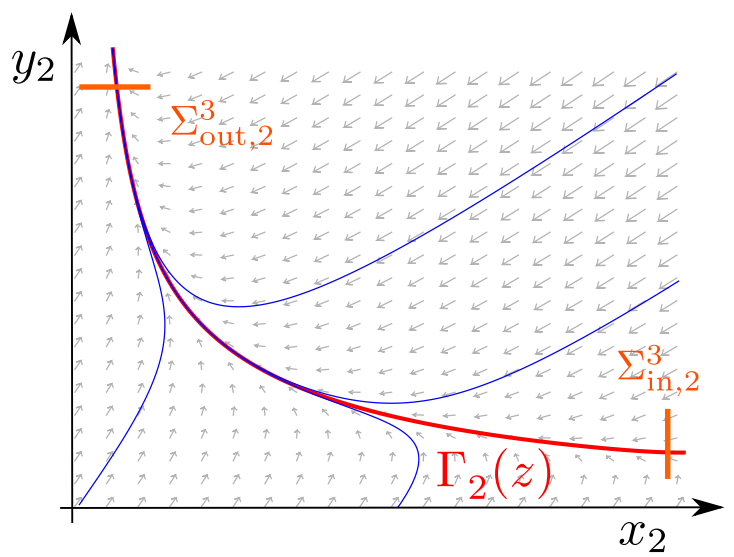

Fig. 4 Phase portrait of (39). There exists a unique orbit with asymptotics (42) and (43) for $x_{2} \rightarrow \pm \infty$. The sections $\Sigma_{\text {in, } 2}^{3}$ and $\Sigma_{\text {out }, 2}^{3}$ transverse to $\Gamma_{2}(z)$ are used in the proof.

see Lemma 2 and (27). Inserting (36) into these expressions gives

$$
\begin{aligned}
& y_{2} \approx x_{2}^{-1}(z+\beta), \\
& x_{2} \approx y_{2}^{-1} z,
\end{aligned}
$$

coinciding with (41) and (40), respectively. This formal argument provides some intuition into why orbits along $\mathcal{S}_{\epsilon}^{2}$ move towards $\mathcal{S}_{\epsilon}^{4}$ (by following the surface $\left\{\Gamma_{2}(z) \mid z \in[0, \infty)\right\}$ in the scaled variables $\left.\left(x_{2}, y_{2}, z\right)\right)$ once reaching a vicinity of $\mathcal{L}^{3}$. Furthermore, if we consider a section $\Sigma_{\text {in }}^{3}=\{x=$ $\left.\sqrt{\epsilon} \xi^{-1},(y / \sqrt{\epsilon}, z) \in \mathcal{R}^{2}\right\}$ then we can, by working in the scaled variables $\left(x_{2}, y_{2}, z\right)$ and equations (38), track initial conditions close to $\Gamma_{2}(z)$ up to a section $\Sigma_{\text {out }}^{3} \subset\left\{y=\sqrt{\epsilon} \xi^{-1},(x / \sqrt{\epsilon}, z) \in \mathcal{R}^{4}\right\}$ using regular perturbation theory. We illustrate the sections $\Sigma_{\text {in/out }}^{3}$ in Fig. 4 using a projection onto the $x_{2} y_{2}$-plane. But this leaves a gap between $\Sigma_{\text {out }}^{2}$ and $\Sigma_{\text {in }}^{3}$. There is a similar gap between $\Sigma_{\text {out }}^{3}$ and $\Sigma_{\text {in }}^{4} \subset\{y=\xi\}$. In the following, to cover these gaps, we provide rigorous mathematical justification for inserting (36) into (42) and (43) (recall that $H$ and $G$ are only defined on $(x, z) \in \mathcal{V}$ and $(y, z) \in \mathcal{W}$ which are fixed with respect to $\epsilon$ ) and extend the slow manifolds into the scaled $\left(x_{2}, y_{2}, z\right)$-system. Henceforth, we put $\Gamma_{2}^{3}=\Gamma_{2}\left(z^{(3)}\right)$, where $z^{(3)}$ is the $z$-value for the point $\gamma^{3}$. 
4.2 Blowup of $\mathcal{L}^{3}$

Consider the extended system of (16)

$$
\begin{aligned}
x^{\prime} & =-x y+\epsilon z-\epsilon \alpha x \\
y^{\prime} & =-x y+\epsilon \beta(1-y)+\epsilon z \\
z^{\prime} & =x y-\epsilon(1+\delta) z \\
\epsilon^{\prime} & =0
\end{aligned}
$$

in the phase space $(x, y, z, \epsilon) \in \mathcal{O} \times\left[0, \epsilon_{0}\right], \epsilon_{0}>0$. The manifolds $\mathcal{S}_{0}^{2}, \mathcal{S}_{0}^{4}, \mathcal{L}^{3}$ are now viewed as subsets of $\mathcal{O} \times\{0\}$. Similarly, $\Gamma_{0} \subset \mathcal{O} \times\{0\}$. We then follow the blowup approach in the formulation of Krupa and Szmolyan Krupa and Szmolyan [2001a] and view (36) as a local version of the blowup transformation

$$
(r, z,(\bar{x}, \bar{z}, \bar{\epsilon})) \mapsto\left\{\begin{array}{l}
x=r \bar{x} \\
y=r \bar{y} \\
z=z \\
\epsilon=r^{2} \bar{\epsilon}
\end{array},\right.
$$

where

$$
(r,(\bar{x}, \bar{y}, \bar{\epsilon})) \in[0, \infty) \times S^{2},
$$

and

$$
S^{2} \equiv\left\{(\bar{x}, \bar{y}, \bar{\epsilon}) \in \mathbb{R}^{3} \mid \bar{x}^{2}+\bar{y}^{2}+\bar{\epsilon}^{2}=1\right\},
$$

is the unit sphere. The variable $z$ remains unchanged under (45) so we keep using this symbol henceforth. The blowup and the analysis in the following resembles the blowup of the planar transcritical bifurcation, see Krupa and Szmolyan [2001b].

Clearly (45) is a diffeomorphism for $r>0$. But the pre-image of $\mathcal{L}^{3}: x=$ $y=\epsilon=0, z \in[0, \infty)$ is a cylinder $(z,(\bar{x}, \bar{y}, \bar{\epsilon})) \in[0, \infty) \times S^{2}$. We therefore say that the transformation (45) (or, actually, the inverse process) blows up $\mathcal{L}^{3}$ to a cylinder. See illustration in Fig. 5.

Notice that the weights on $r$ in (45): 1, 1,0,2, correspond to the weights in (36) since $\epsilon=r_{2}^{2}$. These are the correct weights due to the following. Let $\bar{X}$ denote the vector-field on $(r,(\bar{x}, \bar{y}, \bar{\epsilon})) \in[0, \infty) \times S^{2}$ obtained from transforming (44) by application of (45). Then with the weights in (45), we have $\bar{X}=r \widehat{X}$ with $\widehat{X}$ being well-defined and non-trivial $\left.\widehat{X}\right|_{r=0} \neq 0$, see e.g. Krupa and Szmolyan [2001b]. It is $\widehat{X}$ that we shall study for $r \geq 0$. Notice that for $r>0$, going from $\bar{X}$ to $\widehat{X}$ corresponds to a transformation of time (see e.g. proof of Lemma 1 where apply a similar division of the right hand side). But for $r=0, \bar{X}=0$ whereas $\widehat{X} \neq 0$. The advantage of working with $\widehat{X}$ is therefore that it has improved hyperbolicity properties. Loosely speaking, the division by $r$ divides out/amplifies a vanishing eigenvalue and this process is called desingularization, see e.g. Krupa and Szmolyan [2001a]. 


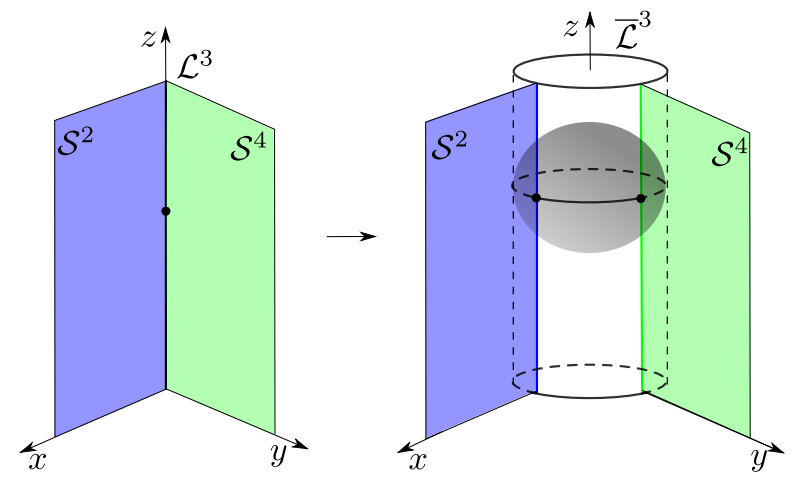

Fig. 5 Blowup of $\mathcal{L}^{3}$ to a line of spheres.

As described in Krupa and Szmolyan [2001a], the coordinates $\left(x_{2}, y_{2}, r_{2}\right)$ in (36) are local coordinates of a directional chart $\kappa_{2}$, obtained by setting $\bar{\epsilon}=1$ on the right hand side of (45):

$$
\left.\begin{array}{c}
r \bar{x}=r_{2} x_{2} \\
r \bar{y}=r_{2} y_{2} \\
r^{2} \bar{\epsilon}=r_{2}^{2}
\end{array}\right\} \Rightarrow(r,(\bar{x}, \bar{y}, \bar{\epsilon})) \mapsto\left\{\begin{array}{l}
x_{2}=\bar{\epsilon}^{-1 / 2} \bar{x} \\
y_{2}=\bar{\epsilon}^{-1 / 2} \bar{y} \\
r_{2}=r \sqrt{\bar{\epsilon}}
\end{array}\right.
$$

This gives (36) as a local form of (45) in the chart $\kappa_{2}$. (37) is therefore also a local form of $\bar{X}$. By division of the right hand side by $r_{2}$, we obtain (38) from (37) which is (up to a further invertible time transformation) a local form of $\widehat{X}$.

The chart $\kappa_{2}$ covers $\bar{\epsilon}>0$ of $S^{2}$. To cover $\bar{x}>0$ and $\bar{y}>0$ we similarly insert $\bar{x}=1$ and $\bar{y}=1$ in (45) to obtain the directional charts $\kappa_{1}$ and $\kappa_{3}$. Denoting the corresponding coordinates by $\left(r_{1}, y_{1}, \epsilon_{1}\right)$ and $\left(x_{3}, r_{3}, \epsilon_{3}\right)$, respectively, we obtain the following local forms of (45):

$$
\begin{array}{ll}
\kappa_{1}: & x=r_{1}, \quad y=r_{1} y_{1}, \quad \epsilon=r_{1}^{2} \epsilon_{1}, \\
\kappa_{3}: & x=r_{3} x_{3}, \quad y=r_{3}, \quad \epsilon=r_{3}^{2} \epsilon_{3},
\end{array}
$$

The change of coordinates between $\kappa_{2}$ and $\kappa_{1}$ is given by

$$
\kappa_{21}:\left(r_{1}, y_{1}, \epsilon_{1}\right) \mapsto\left\{\begin{array}{l}
r_{2}=r_{1} \sqrt{\epsilon_{1}} \\
x_{2}=1 / \sqrt{\epsilon_{1}} \\
y_{2}=y_{1} / \sqrt{\epsilon_{1}}
\end{array},\right.
$$

for $\epsilon_{1}>0$. Similarly, the change of coordinates between $\kappa_{2}$ and $\kappa_{3}$ is given by

$$
\kappa_{23}:\left(r_{3}, x_{3}, \epsilon_{3}\right) \mapsto\left\{\begin{array}{l}
r_{2}=r_{3} \sqrt{\epsilon_{3}} \\
x_{2}=x_{3} / \sqrt{\epsilon_{3}} \\
y_{2}=1 / \sqrt{\epsilon_{3}}
\end{array}\right.
$$


for $\epsilon_{3}>0$. In the charts $\kappa_{1}$ and $\kappa_{3}$, we obtain local versions of $\widehat{X}$ by division of the right hand sides by $r_{1,3}$, respectively. We will follow the standard convention that any object $O$ in the extended space $(x, y, z, \epsilon)$ will be denoted by $\bar{O}$ in the blown up space. In the charts, this object will be given a subscript $O_{i}$.

4.3 Dynamics in chart $\kappa_{1}$

Inserting (46) into (44) gives

$$
\begin{aligned}
\dot{r}_{1} & =r_{1}\left(-y_{1}+\epsilon_{1} z-r_{1} \epsilon_{1} \alpha\right) \\
\dot{y}_{1} & =-y_{1}+\epsilon_{1}\left(\beta\left(1-r_{1} y_{1}\right)+z\right)-\left(-y_{1}+\epsilon_{1} z-r_{1} \epsilon_{1} \alpha\right) y_{1}, \\
\dot{z} & =r_{1}\left(y_{1}-\epsilon_{1}(1+\delta) z\right) \\
\dot{\epsilon}_{1} & =-2 \epsilon_{1}\left(-y_{1}+\epsilon_{1} z-r_{1} \epsilon_{1} \alpha\right)
\end{aligned}
$$

upon division of the right hand side by $r_{1}$. Notice that both $\left\{r_{1}=0\right\}$ and $\left\{\epsilon_{1}=0\right\}$ are invariant for (50). Along their intersection $\left\{r_{1}=\epsilon_{1}=0\right\}$ we obtain the following dynamics

$$
\begin{aligned}
\dot{y}_{1} & =-y_{1}+y_{1}^{2}, \\
\dot{z} & =0 .
\end{aligned}
$$

We therefore obtain two lines of equilibria:

$$
\begin{aligned}
& l_{1}^{2}=\left\{\left(r_{1}, y_{1}, z, \epsilon_{1}\right) \mid y_{1}=0, r_{1}=\epsilon_{1}=0, z \in[0, \infty)\right\}, \\
& l_{1}^{f}=\left\{\left(r_{1}, y_{1}, z, \epsilon_{1}\right) \mid y_{1}=1, r_{1}=\epsilon_{1}=0, z \in[0, \infty)\right\} .
\end{aligned}
$$

For the flow on $\left\{r_{1}=\epsilon_{1}=0\right\}$, described by (51), we see that these two lines are normally hyperbolic: The linearization of (51) about any point in $l_{1}^{2}\left(l_{1}^{f}\right)$ gives -1 (1, respectively) as a single non-zero eigenvalue. Hence $l_{1}^{2}$ is attracting and $l_{1}^{f}$ is repelling. Within $\epsilon_{1}=0$ we find

$$
\begin{aligned}
\dot{r}_{1} & =-r_{1} y_{1}, \\
\dot{y}_{1} & =-y_{1}+y_{1}^{2}, \\
\dot{z} & =r_{1} y_{1},
\end{aligned}
$$

This system has two invariant planes $\mathcal{S}_{1}^{2}$ at $y_{1}=0$, emanating from the line $l_{1}^{3}$, and $\mathcal{F}_{1}$ at $y_{1}=1$, emanating from the line $l_{1}^{f}$. Here $\mathcal{S}_{1}^{2}$ and $\mathcal{F}_{1}$ are just $\mathcal{S}^{2}$ and $\mathcal{F}$ written in the present chart. $\mathcal{S}_{1}^{2}$ is therefore also a plane of equilibria but now the linearization along $\mathcal{S}_{1}^{2}$ gives -1 as a single non-zero eigenvalue.

Finally, the dynamics within $r_{1}=0$ is governed by

$$
\begin{aligned}
\dot{y}_{1} & =-y_{1}+\epsilon_{1}(\beta+z)-\left(-y_{1}+\epsilon_{1} z\right) y_{1}, \\
\dot{z} & =0 \\
\dot{\epsilon}_{1} & =2 \epsilon_{1}\left(y_{1}-\epsilon_{1} z\right)
\end{aligned}
$$


Here we recover $l_{1}^{2}$ and $l_{1}^{f}$ but we also obtain additional eigenvalues due to the final equation. For $l_{1}^{f}$ this additional eigenvalue is 2 but it is 0 along $l_{1}^{2}$. Hence there exists a $2 D$ center manifold $\mathcal{C}_{1}^{3}$ of $l_{1}^{2}$ within $r_{1}=0$.

Proposition 1 Fix any large $k_{1}>0$ and $k_{2}>0$. Then for $\xi>0$ sufficiently small we have: There exists a $3 D$ attracting, locally invariant manifold of (50) of the following form

$$
\begin{gathered}
\mathcal{M}_{1}^{2}=\left\{\left(r_{1}, y_{1}, z, \epsilon_{1}\right) \mid y_{1}=\epsilon_{1}\left(z+\beta+\epsilon_{1} m_{1}^{2}\left(z, \epsilon_{1}\right)\right)+r_{1} \epsilon_{1} m_{2}^{2}\left(r_{1}, z, \epsilon_{1}\right),\right. \\
\left.z \in\left[0, k_{1}\right], r_{1} \in\left[0, k_{2}\right], \epsilon_{1} \in[0, \xi]\right\},
\end{gathered}
$$

with $m_{i}^{2}, i=1,2$, smooth. This manifold contains $\mathcal{S}_{1}^{2}=\left\{y_{1}=\epsilon_{1}=0\right\}$ within $\epsilon_{1}=0$ and a unique center manifold

$$
\mathcal{C}_{1}^{3}=\left\{y_{1}=\epsilon_{1}\left(z+\beta+\epsilon_{1} m_{1}^{2}\left(z, \epsilon_{1}\right)\right), z \in\left[0, k_{1}\right], r_{1}=0, \epsilon_{1} \in[0, \xi]\right\},
$$

within $r_{1}=0$. The flow on $\mathcal{C}_{1}^{3}$ is constant in $z$ and increasing in $\epsilon_{1}$.

Proof Existence of $\mathcal{M}_{1}^{2}$ follows from center manifold theory applied to points within $l_{1}^{1}$. Notice that we can "globalise" $\mathcal{M}_{1}^{2}$ in $r_{1}$ due to the plane of equilibria $\mathcal{S}_{1}^{2}$. The remainder of the proof then follows from simple calculations. In particular, $\mathcal{C}_{1}^{3}$ is unique because each point on $l_{1}^{2}$ is saddle-like within $r_{1}=$ $0, z=$ const. due the hyperbolic contraction in $y_{1}$ and the (non-hyperbolic) expansion in $\epsilon_{1}$.

Now, by construction $\epsilon=r_{1}^{2} \epsilon_{1}$ is an invariant for the equations (50). Therefore restricting $\mathcal{M}^{2}$ to $\epsilon=r_{1}^{2} \epsilon_{1}=$ const. gives an extension of the slow manifold $\mathcal{S}_{\epsilon}^{2}$ in the $(x, y, z)$-variables as a locally invariant manifold up to $x=\sqrt{\epsilon / \xi}$. In particular, in the present chart $\kappa_{1}$, this extended slow manifold, $\mathcal{S}_{\epsilon, 1}^{2}$, intersects $\epsilon_{1}=\xi$ in a graph:

$\mathcal{S}_{\epsilon, 1}^{2} \cap\left\{\epsilon_{1}=\xi\right\}:\left\{\begin{array}{l}r_{1}=\sqrt{\epsilon / \xi} \\ y_{1}=\xi\left(z+\beta+\xi m_{1}^{2}(z, \xi)\right)+\sqrt{\epsilon \xi} m_{2}^{2}(\sqrt{\epsilon / \xi}, z, \xi)\end{array}, z \in\left[0, k_{1}\right]\right.$,

which is smoothly $O(\sqrt{\epsilon})$-close to the intersection of $\mathcal{C}_{1}^{3}$ with $\epsilon_{1}=\xi$ :

$$
\mathcal{C}_{1}^{3} \cap\left\{\epsilon_{1}=\xi\right\}:\left\{\begin{array}{l}
r_{1}=0 \\
y_{1}=\xi\left(z+\beta+\xi m_{1}^{2}(z, \xi)\right)
\end{array}, \quad z \in\left[0, k_{1}\right] .\right.
$$


4.4 Dynamics in chart $\kappa_{3}$

Inserting (47) into (44) gives the following equations

$$
\begin{aligned}
\dot{r}_{3} & =r_{3}\left(-x_{3}+\epsilon_{3}\left(\beta\left(1-r_{3}\right)+z\right)\right), \\
\dot{x}_{3} & =-x_{3}+\epsilon_{3} z-r_{3} \epsilon_{3} \alpha x_{3}-\left(-x_{3}+\epsilon_{3}\left(\beta\left(1-r_{3}\right)+z\right)\right) x_{3}, \\
\dot{z} & =r_{3}\left(x_{3}-\epsilon_{3}(1+\delta) z\right), \\
\dot{\epsilon}_{3} & =-2 \epsilon_{3}\left(-x_{3}+\epsilon_{3}\left(\beta\left(1-r_{3}\right)+z\right)\right),
\end{aligned}
$$

upon division of the right hand side by $r_{3}$. The analysis in this chart is similar to the analysis in $\kappa_{1}$. In particular, the line

$$
l_{3}^{4}=\left\{\left(r_{3}, x_{3}, z, \epsilon_{3}\right) \mid r_{3}=x_{3}=\epsilon_{3}, z \in[0, \infty)\right\},
$$

is partially hyperbolic. By center manifold theory we obtain the results in the following proposition.

Proposition 2 There exists a $3 D$ attracting, locally invariant manifold of (55) of the following form

$$
\begin{gathered}
\mathcal{M}_{3}^{4}=\left\{\left(r_{3}, x_{3}, z, \epsilon_{3}\right) \mid x_{3}=\epsilon_{3}\left(z+\epsilon_{3} m_{1}^{4}\left(z, \epsilon_{3}\right)\right)+r_{3} \epsilon_{3} m_{2}^{4}\left(r_{3}, z, \epsilon_{3}\right),\right. \\
\left.z \in\left[0, k_{1}\right], r_{3} \in\left[0, k_{2}\right], \epsilon_{3} \in[0, \xi]\right\},
\end{gathered}
$$

with $m_{i}^{4}, i=1,2$, smooth. $\mathcal{M}_{3}^{4}$ contains $\mathcal{S}_{3}^{4}=\left\{x_{3}=\epsilon_{3}=0\right\}$ within $\epsilon_{3}=0$ and a non-unique center manifold

$$
\mathcal{C}_{3}^{3}=\left\{x_{3}=\epsilon_{3}\left(z+\epsilon_{3} m_{1}^{4}\left(z, \epsilon_{3}\right)\right), z \in\left[0, k_{1}\right], r_{3}=0, \epsilon_{3} \in[0, \xi]\right\}
$$

within $r_{3}=0$. The flow on $\mathcal{C}_{3}^{3}$ is constant in $z$ and $\epsilon_{3}$ decreases.

Proof Result again follows from center manifold theory and simple calculations. But now $\mathcal{C}_{3}^{3}$ is non-unique because each point on $l_{3}^{4}$ is node-like within $r_{3}=0, z=$ const. due the strong (hyperbolic) contraction in $x_{3}$ and the weak (non-hyperbolic) contraction in $\epsilon_{1}$.

Again, by construction $\epsilon=r_{3}^{2} \epsilon_{3}$ is an invariant for the equations (50). Therefore restricting $\mathcal{M}^{4}$ to $\epsilon=r_{3}^{2} \epsilon_{3}=$ const. gives an extension of the slow manifold $\mathcal{S}_{\epsilon}^{4}$ in the $(x, y, z)$-variables as a locally invariant manifold up to $y=\sqrt{\epsilon / \xi}$. In particular, in the present chart $\kappa_{3}$, this extended slow manifold, $\mathcal{S}_{\epsilon, 3}^{4}$, intersects $\epsilon_{3}=\xi$ smoothly $O(\sqrt{\epsilon})$-close to the intersection of $\mathcal{C}_{3}^{3}$ with $\epsilon_{3}=\xi$.

We illustrate the dynamics of the blowup system in Fig. 6. Upon blowup we have obtained a new singular orbit including $\bar{\Gamma}^{3}=\bar{\Gamma}\left(z^{(3)}\right)$ with improved hyperbolicity properties. 


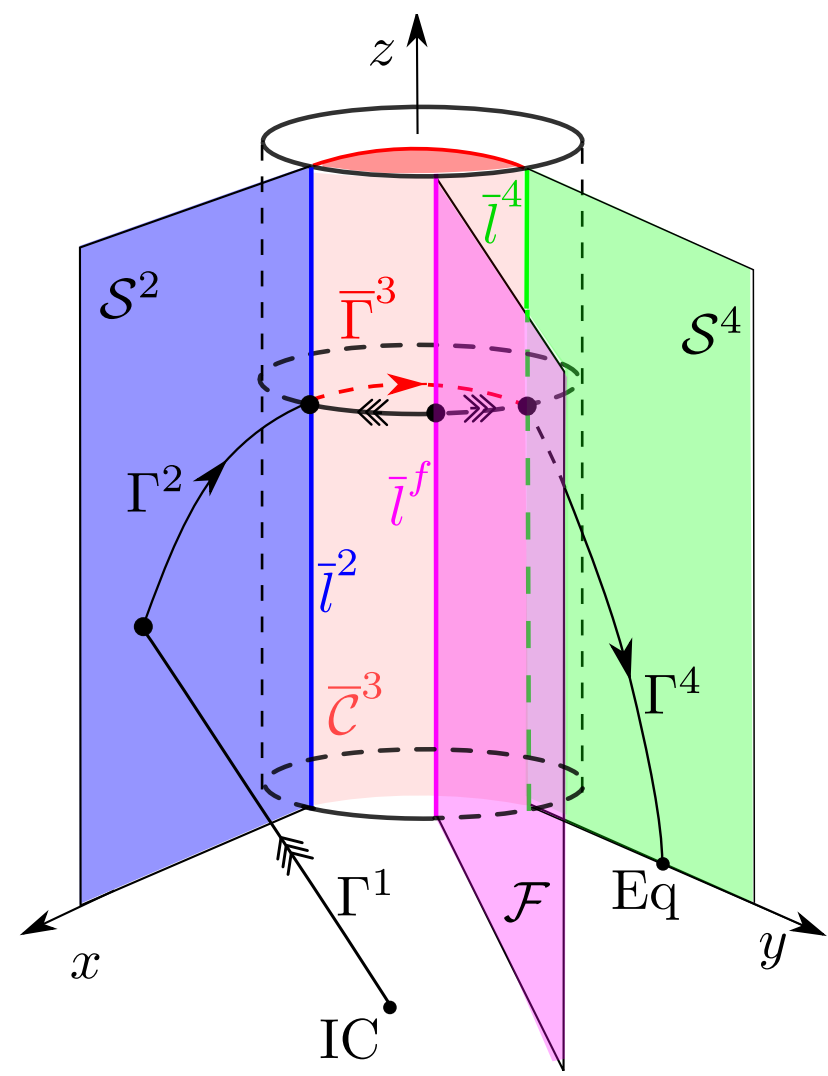

Fig. 6 Blowup dynamics and improved singular orbit.

4.5 Putting things together to complete the proof of Theorem 1

Now, using the coordinate changes (48) and (49), we obtain locally invariant manifolds $\kappa_{21}\left(\mathcal{C}_{1}^{3}\right)$ and $\kappa_{21}\left(\mathcal{C}_{3}^{3}\right)$ within $r_{2}=0$ in chart $\kappa_{2}$ with the following asymptotics:

$$
\begin{aligned}
& y_{2} \approx x_{2}^{-1}(z+\beta), \\
& x_{2} \approx y_{2}^{-1} z,
\end{aligned}
$$

for $x_{2} \rightarrow \infty$ and $y_{2} \rightarrow \infty$, respectively. Now, $\Gamma_{2}(z)$ connects (56) with (57) and therefore (by uniqueness) extends $\kappa_{21}\left(\mathcal{C}_{1}^{3}\right)$ under the forward flow of (39) to a smooth $2 D$ globally invariant manifold $\mathcal{C}_{2}^{3}=\left\{\Gamma_{2}(z), z \in[0, \infty)\right\}$. In fact, we fix the non-unique local manifold $\mathcal{C}_{3}^{3}$ in chart $\kappa_{3}$ such that $\kappa_{23}\left(\mathcal{C}_{3}^{3}\right)$ belongs to this global manifold $\mathcal{C}_{2}^{3}$.

Now, by following $\mathcal{C}_{2}^{3}$, we can guide the manifold $\mathcal{M}_{2}^{2}=\kappa_{21}\left(\mathcal{M}_{1}^{2}\right)$ and flow this forward to obtain an extended manifold $\mathcal{M}_{2}^{2, *}$ in such a way that the nonunique $\mathcal{M}_{2}^{4}$ from chart $\kappa_{3}$ contains the resulting manifold $\mathcal{M}_{2}^{2, *}$. Restricting 
to $\epsilon=$ const. and blowing back down to $(x, y, z)$-space we obtain an invariant manifold containing (by choice) $\mathcal{S}_{\epsilon}^{2}$ and $\mathcal{S}_{\epsilon}^{4}$ where these are defined. This manifold is smooth on $\overline{\mathcal{O}}$ (because it is smooth in the charts $\kappa_{1}, \kappa_{2}$ and $\kappa_{3}$ ) and by regular perturbation theory it is smoothly $O(\sqrt{\epsilon})$-close to $\mathcal{C}_{2}^{3}$ (since it is so at $x_{2}=\xi^{-2}$ or $\epsilon_{1}=\xi$ cf. (53) and (54)) within any compact subset of $\left(x_{2}, y_{2}, z\right)$-space. Parts (A) and (B) of the main theorem now follow. In fact, working in the entry chart $\kappa_{1}$, we realise that $\Gamma_{\epsilon}$ is smoothly $O(\epsilon)$-close to $\Gamma^{(2)}$ at $\Sigma_{\text {out }}^{2} \subset\{x=\xi\}$ (this is just Fenichel), and smoothly $O(\sqrt{\epsilon})$-close at $\Sigma_{\text {in }}^{3} \subset\{x=\sqrt{\epsilon / \xi}\}$ for $\xi>0$ but small. Consequently, cf. the analysis in $\kappa_{2}$ and $\kappa_{3}, \Gamma_{\epsilon}$ is also smoothly $O(\sqrt{\epsilon})$-close to $\Gamma^{4}$ at both $\Sigma_{\text {out }}^{3} \subset\{y=\sqrt{\epsilon / \xi}\}$ (see section in Fig. 4) and $\Sigma_{\text {in }}^{4} \subset\{y=\xi\}$.

\section{Numerics}

In this section, we illustrate our main theoretical result, Theorem 1, through numerical computations of (1) in the non-dimensional form (6) for the parameters in Table 1 (or equivalently Table 2). Fig. 7 shows $\Gamma_{\epsilon}$ in the $x y z$-phase space. Here we also illustrate the extended slow manifold (as described in Section 4.5). We simply compute the slow manifold by forward integration of different initial conditions on the approximations of $\mathcal{S}_{\epsilon}^{2}$ and $\mathcal{S}_{\epsilon}^{4}: y \approx \epsilon x^{-1}(z+\beta)$ and $x \approx \epsilon y^{-1} z$, respectively. Notice how $\Gamma_{\epsilon}$ follows the slow manifold. We do not plot $\Gamma_{0}$ because it is so close to $\Gamma_{\epsilon}$, making it difficult to separate the curves on this scale. Instead we include the point $\gamma^{3}$ to indicate the order of accuracy.

In Fig. 8, we have used similar direct methods to compute the invariant manifold $\mathcal{C}_{2}^{3}$ for $(38)$ in the $\left(x_{2}, y_{2}, z\right)$-variables for $r_{2}=0$. Here we also show $\Gamma_{\epsilon}$ (in red) in the $\left(x_{2}, y_{2}, z\right)$-variables along with its approximation $\Gamma_{2}^{3} \subset \mathcal{C}_{2}^{3}$ (in blue).

Finally, in Fig. 9 we do a time plot of $x(t), y(t)$ and $z(t)$ in blue, red and black, respectively, along $\Gamma_{\epsilon}$ together with their approximations due to $\Gamma^{2}$, $\Gamma_{2}^{3}$ and $\Gamma^{4}$ on a time interval near third phase. The dashed blue curve is $x(t)$ along $\Gamma^{2}$, see expression in (34). It is defined up until $T\left(x^{(2)}\right)$. In agreement with our analysis, this curve follows $x(t)$ (in blue) along $\Gamma_{\epsilon}$ outside $\mathbb{P}^{3}$. Inside $\mathbb{P}^{3}$ the separation between the curves is larger. Similarly, the dashed red line is $y(t)$ along $\Gamma^{4}$, see expression in (35), defined for $t \geq T\left(x^{(2)}\right)$. It follows the actual $y(t)$ (in red) along $\Gamma_{\epsilon}$ outside $\mathbb{P}^{3}$. Within $\mathbb{P}^{4}$ the approximation of $\Gamma_{\epsilon}$ by $\Gamma^{4}$ is less accurate. There is also a dashed black curve due to $z(t)$ along $\Gamma^{2}$ and $\Gamma^{4}$, see expressions in (34) and (35) for $t \gtrless T\left(x^{(2)}\right)$, but this curve is inseparable from the full black curve representing $z(t)$ along $\Gamma_{\epsilon}$. Now, the dotted lines in blue, red and black are due to the approximation by $\Gamma_{2}^{3}$, setting $x(t)=\sqrt{\epsilon} x_{2}(t / \sqrt{\epsilon}), x(t)=\sqrt{\epsilon} x_{2}(t / \sqrt{\epsilon}), z(t)=z^{(3)}$, with $x_{2}\left(\tau_{2}\right), y_{2}\left(\tau_{2}\right)$ described in Lemma 3 . This approximation is accurate within $\mathbb{P}^{3}$ as desired but less accurate outside. In the context of matched asymptotics one would refer to the dotted lines as outer solutions and the dashed ones as inner solutions. 


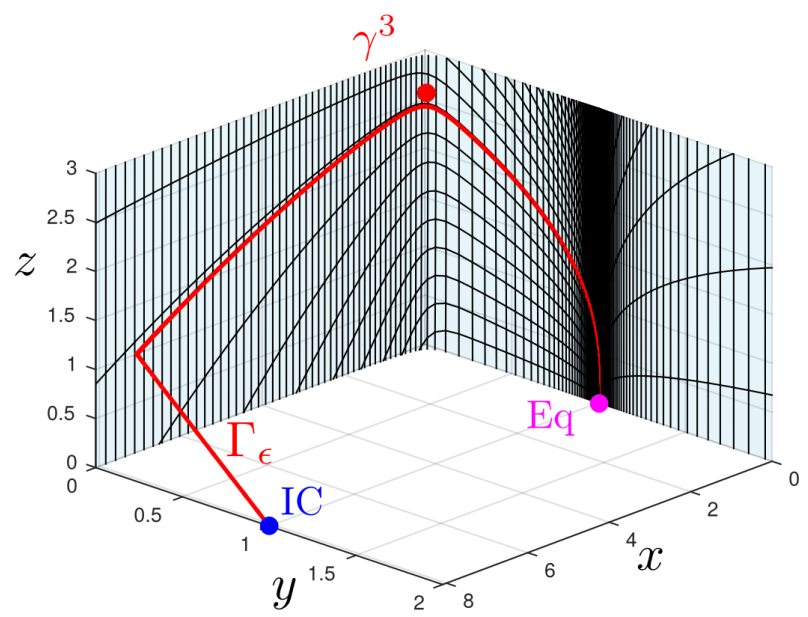

Fig. $7 \Gamma_{\epsilon}$ (red) and the extended slow manifold (surface).

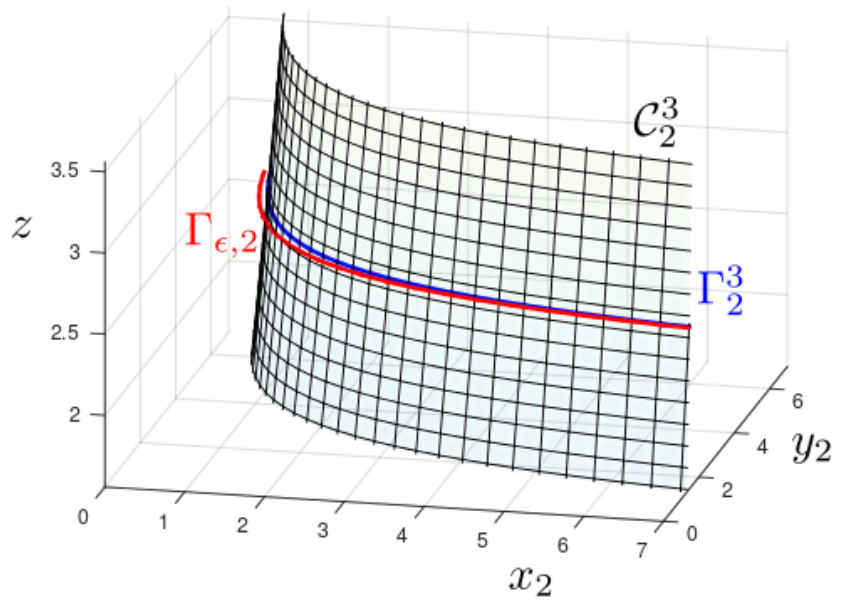

Fig. $8 \Gamma_{\epsilon}$ (red) in the scaled variables $\left(x_{2}, y_{2}, z\right)$, where it by our notation convention is denoted by $\Gamma_{\epsilon, 2}$, and the center manifold $\mathcal{C}_{2}^{3}$ (surface). $\Gamma_{\epsilon, 2}$ is approximated by $\Gamma_{2}^{3} \subset \mathcal{C}_{2}^{3}$ in blue.

\section{Discussion}

Following our analysis, we can attribute each phase with different scales. For example, during phase $\mathbb{P}^{1}$, all variables $x, y, z$ are $O(1)$. During phase $\mathbb{P}^{2}$, 


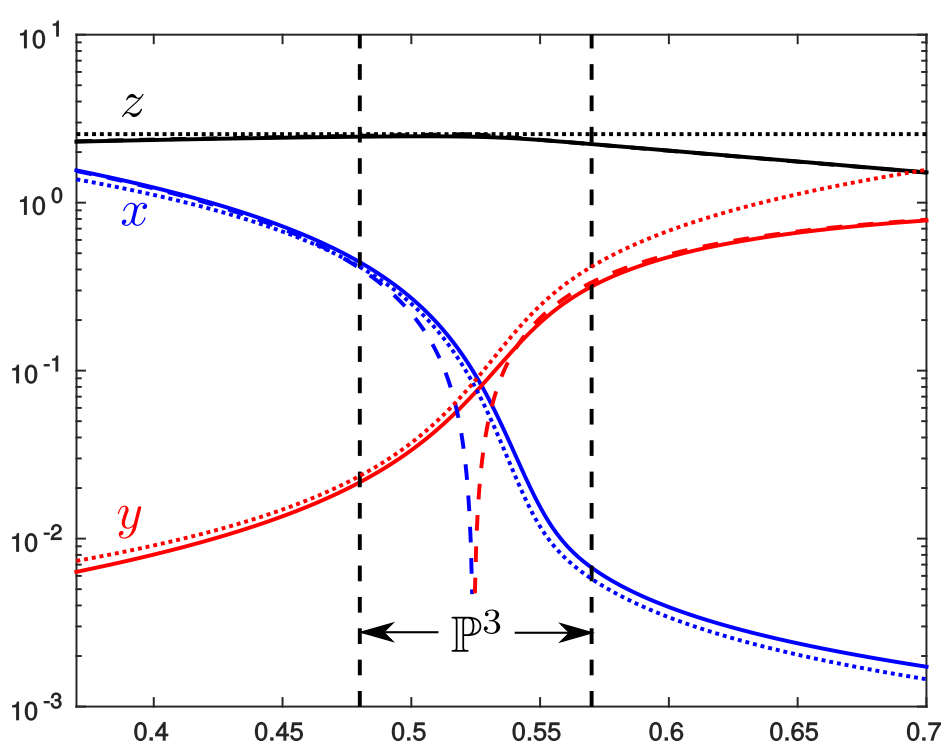

Fig. $9 x(t)$ (blue), $y(t),($ red $), z(t)$ (black) along $\Gamma_{\epsilon}$. The dashed lines are approximations (outer solutions) due to $\Gamma^{2}$ and $\Gamma^{4}$ whereas the dotted lines are approximations (inner solutions) due to $\Gamma_{2}^{3}$.

described by motion along the slow manifold $\mathcal{S}_{\epsilon}^{2}, y=O(\epsilon)$. Along this phase $x$ is monotonically decreasing since $x$ is so along the reduced flow on $\mathcal{S}^{2}$. But then since $y \approx \epsilon x^{-1}(z+\beta)$ within $\mathcal{S}_{\epsilon}^{2}, y$ is slightly increasing. Once $x$ reaches $O(\sqrt{\epsilon})$ then $y=O(\sqrt{\epsilon})$ and we enter phase $\mathbb{P}^{3}$. Here $x, y=O(\sqrt{\epsilon})$ throughout and $\mathbb{P}^{3}$ is described by the solution curve $\Gamma_{2}^{3}$ in the scaled variables $x_{2}=x / \sqrt{\epsilon}, y_{2}=y / \sqrt{\epsilon}$ (recall Fig. 4). $z$ is almost constant but $x_{2}$ and $y_{2}$ vary quickly in agreement with the initial observations by Peletier and Gabrielsson [2012]. At the end of phase $\mathbb{P}^{3}$, we have $x \approx \epsilon y^{-1} z$ and therefore as $y$ increases to $O(1)$ we have $x=O(\epsilon)$ during the final phase $\mathbb{P}^{4}$.

Remark 5 In Peletier and Gabrielsson $[2012,2013]$, the third phase is described by the scaling

$$
\tilde{x}=\frac{k_{\text {off }}}{k_{\text {on }}} u
$$

see e.g. [Peletier and Gabrielsson, 2013, Eq. (5.17)]. By Table 2, it follows that

$$
x=\epsilon u .
$$

This scaling therefore zooms in on $x=0$ for $0<\epsilon \ll 1$. Inserting (58) into (16) gives

$$
\begin{aligned}
& u^{\prime}=-u y+z-\epsilon \alpha u, \\
& y^{\prime}=\epsilon(-u y+\beta(1-y)+z), \\
& z^{\prime}=\epsilon(u y-(1+\delta) z),
\end{aligned}
$$


which is a standard slow-fast system with $u$ being fast and $y, z$ being slow. Setting $\epsilon=0$, gives $u=y^{-1} z$ as an attracting critical manifold for $y>0$. Notice by (58), $x \approx \epsilon y^{-1} z$. Referring to Lemma 2 , in particular the expression (29), we realise that the critical manifold is just $\mathcal{S}_{\epsilon}^{4}$ to leading order written in the $(u, y, z)$-coordinates. This scaling therefore only describes phase $\mathbb{P}^{4}$, not phase $\mathbb{P}^{3}$. Indeed, the manifold $u=y^{-1} z$ is not compact for $y>0$ and the approximation by Fenichel therefore breaks down as $y$ approaches 0 (which is the relevant regime for $\mathbb{P}^{3}$ ).

Dependency on $x_{0}$. For $x_{0} \rightarrow \infty$ the duration of $\mathbb{P}^{1}$ diminishes, see e.g. Appendix $\mathrm{B}$ and (72) where we have multiplied the right hand side by $u=x^{-1}$ to slow down time. On the other hand, the duration of $\mathbb{P}^{2}$ enhances and $(x(t), y(t), z(t))$ spends an increasing amount of time close to the invariant line $x>0, y=0, z=\delta^{-1} \beta$ of (23). We can also see this in the expression for $\Gamma^{2}$, see (34), as $T\left(x^{(2)}\right) \rightarrow \infty$ when $x_{0} \rightarrow \infty$.

On the other hand, the phase $\mathbb{P}^{2}$ diminishes as $x_{0} \rightarrow 0^{+}$. Indeed, for $x_{0}<1$ then $\Gamma_{\epsilon}$ converges directly to a point on $\mathcal{S}_{\epsilon}^{4}$ and hence there are no second and third phase. In this situation $\Gamma_{\epsilon}$ is described by Fenichel's theory near $\mathcal{S}^{4}$ and $\lim _{\epsilon \rightarrow 0^{+}} \Gamma_{\epsilon}$ is the union of

$$
\Gamma^{1}=\left\{(x, y, z) \in \mathcal{O} \mid x=x_{0}-s, y=1-s, z=s, s \in\left[0, x_{0}\right)\right\}
$$

and

$$
\Gamma^{4}=\left\{(x, y, z) \in \mathcal{O} \mid x=0, y(t)=1+\left(1-x_{0}-1\right) e^{-\beta t}, z=x_{0} e^{-\delta t}, t \geq 0\right\}
$$

Here $\Gamma^{1}$ is the forward orbit of IC under the flow of the layer problem (21). It is asymptotic to $(x, y, z)=\left(0,1-x_{0}, x_{0}\right)$. From here $\Gamma^{4}$ is the forward orbit of the reduced problem on $\mathcal{S}^{4}(24)$. An example is illustrated in Fig. 3 (orange curve).

Dependency on other parameters. The dependency on all other parameters can easily be derived from the explicit expression of $\Gamma_{0}$ or simply from the reduced problems on $\mathcal{S}^{2}$ and $\mathcal{S}^{4}$, see (23) and (24). From (23), for example, we realise that decreasing (increasing) $\beta$ has the following affect on $\mathbb{P}^{2}$ : it (a) increases (decreases) the time $T\left(x^{(2)}\right)$ and (b) decreases (increases, respectively) the $z$-value, $z^{(3)}$, of $\gamma^{3}$. For $\mathbb{P}^{4}$, increasing (decreasing) $\beta$ implies by (24) that $y$ converges faster (slower, respectively) to its steady-state value $y=1$. Similarly, decreasing (increasing) $\alpha$ increases (decreases, respectively) $T\left(x^{(2)}\right)$ during $\mathbb{P}^{2}$ but to leading order $z$ is unaffected. Also $\alpha$ has no affect on $\mathbb{P}^{4}$ to leading order. Moreover, by decreasing (increasing) $\delta, z$ and $z^{(3)}$ both increase (decrease) during phase $\mathbb{P}^{2}$ while for $\mathbb{P}^{4}$ the convergence rate of $z$ towards its steadystate value $z=0$ is enhanced (detracted, respectively). Finally, to leading order only $\beta$ affects $\mathbb{P}^{3}$. 
Estimation of the parameters. By dividing the data of the evolution of the concentrations into the separate phases, one can approximate the parameters. An approach is described in Peletier and Gabrielsson [2013]. Following our main theorem, we can provide an alternative estimation process through a data set $(\tilde{x}, \tilde{y}, \tilde{z})(\tilde{t}), \tilde{t} \geq 0$, as follows, using the definitions of the non-dimensional numbers in Table 2 :

(i) $\tilde{y}_{0}$ is estimated by $\tilde{y}_{0} \approx y\left(t_{1}\right)$ with $t_{1} \gg 0$ large or by $\tilde{x}^{2}=\tilde{x}_{0}-\tilde{y}_{0}$ using an estimate of $\tilde{x}^{2}$, the value of $\tilde{x}$ at the end of the first phase;

(ii) $\epsilon$ can be estimated from a point $\left(x\left(t_{i i}\right), y\left(t_{i i}\right), z\left(t_{i i}\right)\right)$ during $\mathbb{P}^{4}$ by the condition that $\left(x\left(t_{i i}\right), y\left(t_{i i}\right), z\left(t_{i i}\right)\right) \in \mathcal{S}_{\epsilon}^{4}$ :

$$
\tilde{x}\left(t_{i i}\right) \approx \tilde{y}_{0} \epsilon \tilde{y}\left(t_{i i}\right)^{-1} \tilde{z}\left(t_{i i}\right),
$$

obtained from (30) using (5) and multiplication of $\tilde{y}_{0}$;

(iii) $k_{\text {out }}=\beta k_{\text {off }}$ is the slope of $-\log \left(\tilde{y}_{0}-\tilde{y}(\tilde{t})\right)$ during $\mathbb{P}^{4}$, see $(24)$;

(iv) Given $\tilde{y}_{0}$ and $\epsilon$ from (i) and (ii), respectively, $\beta$ can be determined directly from $\mathbb{P}^{2}$ by the condition that $\left(x\left(t_{i v}\right), y\left(t_{i v}\right), z\left(t_{i v}\right)\right) \in \mathcal{S}_{\epsilon}^{2}$ :

$$
\tilde{y}\left(t_{i v}\right) \approx \tilde{y}_{0} \epsilon \tilde{x}\left(t_{i v}\right)^{-1}\left(\tilde{z}\left(t_{i v}\right)+\tilde{y}_{0} \beta\right),
$$

obtained from (29) using (5) and multiplication of $\tilde{y}_{0}$;

(v) $k_{\mathrm{eRL}}=\delta k_{\mathrm{off}}$ is the slope of $-\log \tilde{z}(\tilde{t})$ during $\mathbb{P}^{4}$, see $(24)$;

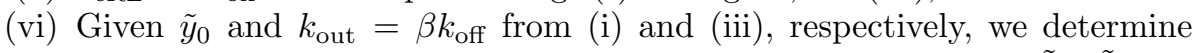
$k_{\mathrm{eL}}=\alpha k_{\text {off }}$ as follows: Let $\tilde{x}_{1}$ and $\tilde{x}_{2}<\tilde{x}_{1}$ be two values of $\tilde{x}$ at $\tilde{t}=\tilde{t}_{1}$ and $\tilde{t}=\tilde{t}_{2}>\tilde{t}_{1}$ during phase $\mathbb{P}^{2}$. Then by (25) and (5) we obtain the following equation for $\tilde{t}_{2}-\tilde{t}_{1}$ :

$$
\tilde{t}_{2}-\tilde{t}_{1}=-\frac{\ln \left(\frac{\tilde{y}_{0}^{-1} \tilde{x}_{2}+\alpha^{-1} \beta}{\tilde{y}_{0}^{-1} \tilde{x}_{1}+\alpha^{-1} \beta}\right)}{\alpha k_{\mathrm{off}}}=-\frac{\ln \left(\frac{\tilde{y}_{0}^{-1} \tilde{x}_{2}+k_{\mathrm{eL}}^{-1} k_{\mathrm{out}}}{\tilde{y}_{0}^{-1} \tilde{x}_{1}+k_{\mathrm{eL}}^{-1} k_{\mathrm{out}}}\right)}{k_{\mathrm{eL}}} .
$$

Having determined $k_{\text {out }}$ and $\beta$ in (iii) and (iv), respectively, we directly obtain

$$
k_{\mathrm{off}}=\beta^{-1} k_{\mathrm{out}}
$$

by Table 2. Furthermore, by step (i) we have

$$
k_{\text {in }}=k_{\text {out }} \tilde{y}_{0},
$$

using (2). Finally, step (ii) implies that

$$
k_{\mathrm{on}}=\frac{k_{\mathrm{off}}}{\tilde{y}_{0} \epsilon},
$$

obtained by rearranging the equation for $\epsilon$ in Table 2 . The quantities on the right hand side of (65) are at this stage of the procedure all known. In this way, we therefore approximate all six $k_{i}, i=$ off, on, eL, eRL, in and out, dimensional numbers. The error of these estimates can easily be estimated because they are based on equations that in non-dimensional form are correct up to $\mathcal{O}(\epsilon)$. Also the error of this approach should, by Theorem 2 and the fact 
that we do not use phase $\mathbb{P}^{1}$ in (i)-(vi), be largely independent of the initial dose $\tilde{x}_{0}>\tilde{y}_{0}$.

Notice in comparison with Peletier and Gabrielsson [2013], that this process does not use the third, nonlinear phase $\mathbb{P}^{3}$. Also, $k_{\text {on }}$ is estimated in a different way in Peletier and Gabrielsson [2013] using an estimate of the half-time of the contraction towards $\mathcal{S}^{2}$ during $\mathbb{P}^{1}$. Moreover, the authors in Peletier and Gabrielsson [2013] do not exploit the estimates (60) and (61) in step (ii) and step (iv) for the approximation of the slow manifolds $\mathcal{S}_{\epsilon}^{2}$ and $\mathcal{S}_{\epsilon}^{4}$, see (29) and (30).

In the following, we apply the procedure (i)-(vi) to the data in Fig. 1 obtained from a numerical computation using Matlab's ODE-solver ode45 with tolerances $=10^{-9}$. In step (i), we just take the last data point

$$
\tilde{y}_{0}=12.3595 \text {. }
$$

In step (ii), we use linear interpolation of the data points produced by Matlab to obtain $\tilde{x}=0.0095, \tilde{y}=11.9049, \tilde{z}=10.2769$ at $\tilde{t}_{i i}=900$ within $\mathbb{P}^{4}$, see also Fig. 1 (b). This gives

$$
\epsilon=8.9063 \times 10^{-4} .
$$

after rearranging (60). Matlab's detailed output is probably not comparable with a real-life experiment. But our primary aim with this section is to illustrate how our approach can be used and for this purpose the amount of data is not important.

In step (iii), we consider $\tilde{t}=800$ and $\tilde{t}=1000$ within $\mathbb{P}^{4}$ and obtain $\tilde{y}=1.1035$ and $\tilde{y}=0.1876$ using linear interpolation, respectively, see Fig. 1 (b). This gives

$$
k_{\text {out }} \approx-\frac{\log 1.1035-\log 0.1857}{200}=0.008860 .
$$

In step (iv), we take $\tilde{t}_{i v}=100$ within $\mathbb{P}^{2}$ and obtain $\tilde{x}=65.2615, \tilde{y}=0.0216$, $\tilde{z}=18.6222$, see Fig. 1 (a). This gives

$$
\beta \approx 8.8605 \text {, }
$$

after rearranging (61). Then by (63) we obtain

$$
k_{\mathrm{off}} \approx 9.9989 \times 10^{-4} .
$$

In step (v), we again consider $\tilde{t}=800$ and $\tilde{t}=1000$ within $\mathbb{P}^{4}$ and find $\tilde{z}=13.8696$ and $\tilde{z}=7.6145$, respectively, see Fig. 1 (b). This gives

$$
k_{\mathrm{eRL}}=0.002998 .
$$

In step (vi), we finally take $\tilde{t}_{1}=100$ and $\tilde{t}_{2}=200$ and obtain

$$
\tilde{x}_{1}=65.2615, \quad \tilde{x}_{2}=45.9876,
$$


Table 3 Estimation of the parameters using the approach described in (i)-(vi). The maximum relative error is $0.5 \%$, see third row.

\begin{tabular}{|c||c|c|c|c|c|c|}
\hline & $k_{\text {on }}$ & $k_{\text {off }}$ & $k_{\mathrm{eL}}$ & $k_{\text {in }}$ & $k_{\text {out }}$ & $k_{\mathrm{eRL}}$ \\
\hline Value & 0.091 & 0.001 & 0.0015 & 0.11 & 0.0089 & 0.003 \\
\hline Est. val. & 0.09079 & $9.9989 \times 10^{-4}$ & 0.0015030 & 0.1095 & 0.008860 & 0.002998 \\
\hline Rel. err. (\%) & 0.3 & 0.06 & 0.2 & 0.5 & 0.5 & 0.06 \\
\hline
\end{tabular}

see Fig. 1 (a). Inserting this into (62) together with (66) gives a nonlinear equation

$$
100=-\frac{\ln \left(\frac{3.7208+k_{\mathrm{eL}}^{-1} 0.008860}{5.2803+k_{\mathrm{eL}}^{-1} 0.008860}\right)}{k_{\mathrm{eL}}}
$$

for $k_{\mathrm{eL}}$, which we solve using Matlab's fsolve. This gives

$$
k_{\mathrm{eL}}=0.0015030 .
$$

Finally,

$$
k_{\text {in }}=0.1095, \quad k_{\text {on }}=0.09079,
$$

using (64) and (65). We summarise our estimates in Table 3. The maximum relative error of the estimated parameters is $0.5 \%$.

Higher order approximations. It is possible to use our approach and results to obtained higher order approximations of $\Gamma_{\epsilon}$ and capture the $O(\sqrt{\epsilon})$-terms. This is not conceptually difficult (we just do the matching in the charts, see also van Gils et al. [2005]) but the calculations are tedious and the formulas are lengthy. Also we are unsure what extra insight this would provide. We therefore also leave out such results from the present manuscript.

Rebounding. Finally, we conclude the paper by describing rebounding. This is the phenomenon where the receptor levels (concentration of $\tilde{y}$ ) exceeds its baseline value $\tilde{y}_{0}: \tilde{y}(t)>\tilde{y}_{0}$ after application of the drug $\tilde{x}_{0}$. In Aston et al. [2014] the authors show that for (1) rebounding occurs if and only if the elimination rate of the complex product $k_{e R L}$ is slower than the elimination rates of the drug $k_{e L}$ and of the receptor $k_{\text {out }}$ :

$$
k_{\mathrm{eRL}}<\min \left(k_{\mathrm{eL}}, k_{\text {out }}\right),
$$

see [Aston et al., 2014, Corollary 3.2]. In our non-dimensionalised variables, rebounding means that $\Gamma_{\epsilon} \cap\{y>1\}$ is non-empty. By (67) it occurs if and only if

$$
\delta<\min (\alpha, \beta)
$$

In the following, we reproduce this result for $0<\epsilon \ll 1$ using our perturbation approach. For this we first realise by Theorem 1 (A) that rebounding (i.e. 
$y(t)>1)$ can only occur during the final phase $\mathbb{P}^{4}$. This phase occurs on the slow manifold $\mathcal{S}_{\epsilon}^{4}$, see (30). Therefore we can reduce the problem to the two-dimensional system (31), in terms of $y$ and $z$, obtained by inserting $x=$ $\epsilon z H(y, z, \epsilon)$ into (6). Eq is a stable node for these equations for any $0 \leq \epsilon \ll 1$ (recall also Remark 3(b)). In particular, for $\epsilon=0$ we obtain the reduced problem on $\mathcal{S}^{4}$, see (24), in agreement with Fenichel's theory, in which case the linearization about Eq has the following eigenvalues $-\beta$ and $-\delta$ with associated eigenvectors $v_{1}=(1,0)^{T}, v_{2}=(0,1)^{T}$, respectively, in the $y z$-plane. In fact, the set $M_{2}=\{y=1, z \geq 0\}$, obtained by the span of the eigenvector $v_{2}$, is invariant for the flow of (31) for $\epsilon=0$. Similarly, $M_{1}=\{y \geq 0, z=0\}$, obtained by the span of the eigenvector $v_{1}$, is also invariant, even for all $\epsilon \geq 0$. Consider first $\beta<\delta$. Then $v_{1}\left(v_{2}\right)$ is the weak (strong) eigenvector of Eq for $\epsilon=0 . M_{2}$ is therefore the unique strong stable manifold, tangent to $v_{2}$ at Eq; all other orbits of the reduced problem (i.e. $\epsilon=0$ ) on $\mathcal{S}^{4}$ are tangent to $M_{1}$ at Eq. In this case, $\Gamma^{4}$ therefore approaches Eq from below and tangent to $M_{1}$ in such a way that $y$ is monotonically increasing. To perturb this into $0<\epsilon \ll 1$ we note the following simple consequences of Theorem 1 (A) and regular perturbation theory within the compact set $\mathcal{W}$, recall also Lemma 2 and see Fig. 10 for an illustration : (a): $\Gamma_{\epsilon}$ is $\mathcal{O}(\sqrt{\epsilon})$-close to $\Gamma^{4}$ at $\Sigma_{\text {in }}^{4}$ (entry to phase $\left.\mathbb{P}^{4}\right)$; (b): $M_{2}$ perturbs by an $\mathcal{O}(\epsilon)$-amount. Therefore for $0<\epsilon \ll 1$, the projection of $\Gamma_{\epsilon}$ onto the $(y, z)$-plane is contained within the subset of $\mathcal{W}$ bounded by $M_{2}$ and the $z$-axis, consisting of all orbits that approach Eq tangent to the $M_{1}$; in particular $\Gamma_{\epsilon} \cap \Sigma_{\text {in }}^{4}$ is uniformly bounded away from $M_{2}$ for $0<\epsilon \ll 1$. This means that the solution approaches Eq from below and no rebounding occurs.

On the other hand, for $\beta>\delta$ (the situation in Table 1) the vector $v_{2}$ is now the weak eigenvector and hence $\Gamma^{4}$ approaches Eq tangent to $M_{2}$. To determine whether rebounding occurs for $\beta \geq \delta$, we compute the weak eigenvector at Eq on $\mathcal{S}_{\epsilon}^{2}$ to higher order in $\epsilon$. We therefore consider (31) with $0<\epsilon \ll 1$ and compute the linearization of the resulting equations about Eq, setting $(y, z)=(1,0)$. This gives the following eigenvalues

$$
\lambda_{1}=-\beta, \quad \lambda_{2}=-\delta+\epsilon(\delta-\alpha)+O\left(\epsilon^{2}\right),
$$

and the following corresponding eigenvectors

$$
v_{1}=(1,0)^{T}, \quad v_{2}=\left(\epsilon(\beta-\delta)^{-1}(\alpha-\delta)+O\left(\epsilon^{2}\right), 1\right)^{T},
$$

respectively, for $\beta \neq \delta$. For $\beta>\delta$ the eigenvalue $\lambda_{2}$ is weakest for all $0 \leq$ $\epsilon \ll 1$. The projection of $\Gamma_{\epsilon}$ onto the $(y, z)$-plane therefore approaches Eq tangent to the corresponding eigenvector $v_{2}$. Now by (68), for $\alpha>\delta$ we have $(\beta-\delta)^{-1}(\alpha-\delta)>0$ and therefore the span of $v_{2}$ based at Eq intersects the "rebound" region $y>1, z>0$. Hence rebounding does occur in this case for $0<\epsilon \ll 1$. On the other hand, for $\alpha<\delta$, then $(\beta-\delta)^{-1}(\alpha-\delta)<0$ and hence the span of $v_{2}$ based at Eq does not intersects $y>1, z>0$. Therefore, referring again to Theorem 1 (A), we conclude that rebounding does not occur in this 


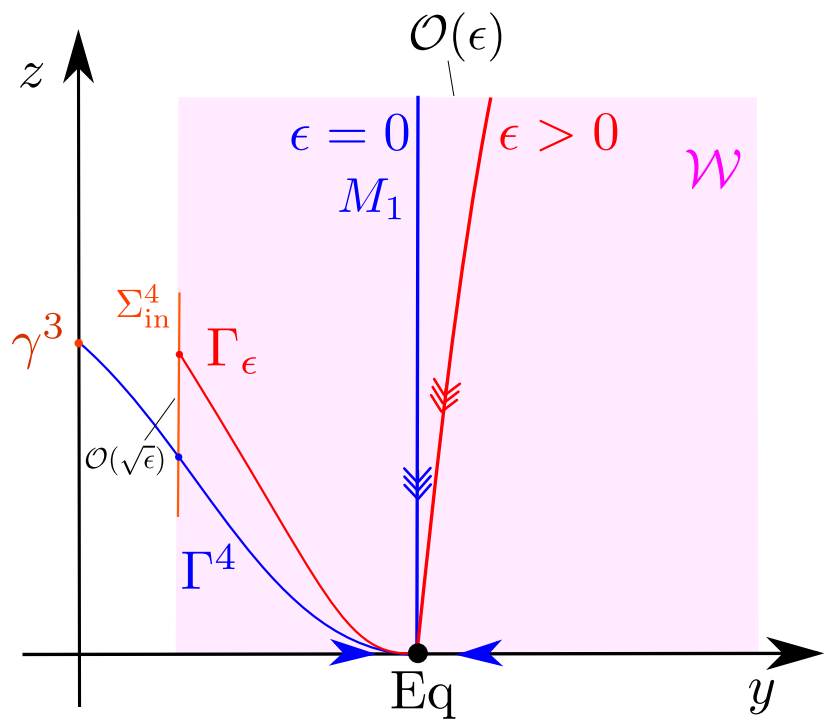

Fig. 10 Illustration of the dynamics on $\mathcal{S}_{\epsilon}^{4}$ within $\mathcal{W}$ during the final phase $\mathbb{P}^{4}$ for $\beta<\delta$ and $0 \leq \epsilon \ll 1 . M_{2}$ is, in this situation, the strong stable manifold (indicated by the tripple-headed arrows). The invariant $y$-axis coincides with the weak eigenspace (indicated by single-headed arrows). By Theorem 1 (A), $\Gamma_{\epsilon}$ is $\mathcal{O}(\sqrt{\epsilon})$-close to $\Gamma^{4}$ at $\Sigma_{\text {in }}^{4}$ and by regular perturbation theory $M_{1}$ perturbs by an $\mathcal{O}(\epsilon)$-amount within the compact set $\mathcal{W}$.

instance. ${ }^{1}$ The remaining cases $\beta=\delta$ and $\alpha=\delta$ have to be handled separately using higher order terms. We leave out the details of these calculations here.

Now, although our approach only works for $0<\epsilon \ll 1$, the singular perturbation theory tackles the global part of the problem relatively easy and therefore reduces the problem to a local one near Eq almost directly. We therefore anticipate that our method might be more useful for more complicated systems of this kind.

\section{Acknowledgement}

The author would like to thank the students Anders Eltved, Sigrun Nordli and Asger Limkilde for their initial work on this problem.

\section{References}

P. J. Aston, G. Derks, A. Raji, B. M. Agoram, and P. H. van der Graaf. Mathematical analysis of the pharmacokinetic-pharmacodynamic (pkpd)

\footnotetext{
1 Notice also that the position of the weak eigenspace in this case implies that $\dot{y}<0$ along $y=1$ for all $z, \epsilon>0$ sufficiently small. In fact, inserting $y=1$ into (31) gives this directly: $\dot{y}=-\epsilon z(\delta-\alpha+\mathcal{O}(\epsilon))$.
} 
behaviour of monoclonal antibodies: Predicting in vivo potency. Journal of Theoretical Biology, 281(1):113-121, 2011.

P. J. Aston, G. Derks, B. M. Agoram, and P. H. van der Graaf. A mathematical analysis of rebound in a target-mediated drug disposition model: I.without feedback. Journal of Mathematical Biology, 68(6):1453-1478, 2014.

E. Bossolini, M. Brøns, and K. U. Kristiansen. Singular limit analysis of a model for earthquake faulting. Nonlinearity, 30(7):2805-2834, 2017.

P. Dua, E. Hawkins, and P. H. Van Der Graaf. A tutorial on target-mediated drug disposition (tmdd) models. 2016.

N. Fenichel. Persistence and smoothness of invariant manifolds for flows. Indiana University Mathematics Journal, 21:193-226, 1971.

A. Goeke, S. Walcher, and E. Zerz. Determining "small parameters" for quasisteady state. Journal of Differential Equations, 259(3):1149-1180, 2015.

C. K. R. T. Jones. Geometric Singular Perturbation Theory, Lecture Notes in Mathematics, Dynamical Systems (Montecatini Terme). Springer, Berlin, 1995.

T. Kaper. An introduction to geometric methods and dynamical systems theory for singular perturbation problems. Proceedings of Symposia in Applied Mathematics, 56, 1999.

I. Kosiuk and P. Szmolyan. Geometric singular perturbation analysis of an autocatalator model. Discrete and Continuous Dynamical Systems - Series $S, 2(4): 783-806,2009$.

I. Kosiuk and P. Szmolyan. Scaling in singular perturbation problems: Blowing up a relaxation oscillator. Siam Journal on Applied Dynamical Systems, Siam J. Appl. Dyn. Syst, Siam J a Dy, Siam J Appl Dyn Syst, Siam Stud Appl Math, 10(4):1307-1343, 2011.

I. Kosiuk and P. Szmolyan. Geometric analysis of the Goldbeter minimal model for the embryonic cell cycle. Journal of Mathematical Biology, J. Math. Biol, J Math Biol, 2015.

I. Kosiuk. Relaxation oscillations in slow-fast systems beyond the standard form. Phd thesis, University of Leipzig, 2012.

M. Krupa and P. Szmolyan. Extending geometric singular perturbation theory to nonhyperbolic points - fold and canard points in two dimensions. SIAM Journal on Mathematical Analysis, 33(2):286-314, 2001.

M. Krupa and P. Szmolyan. Extending slow manifolds near transcritical and pitchfork singularities. Nonlinearity, 14(6):1473, 2001.

C. Kuehn. Multiple Time Scale Dynamics. Springer-Verlag, Berlin, 2015.

G. Levy. Pharmacologic target-mediated drug disposition. Clinical Pharmacology and Therapeutics, 56(3):248-52, 248-252, 1994.

D. E. Mager and W. J. Jusko. General pharmacokinetic model for drugs exhibiting target-mediated drug disposition. Journal of Pharmacokinetics and Pharmacodynamics, 28(6):507-532, 2001.

J. D. Meiss. Differential dynamical systems, volume 14. Society for Industrial and Applied Mathematics, 2007.

D. G. Patsatzis, D. T. Maris, and D. A. Goussi. Asymptotic analysis of a target-mediated drug disposition model: Algorithmic and traditional ap- 
proaches. Bulletin of Mathematical Biology, 78(6):1121-1161, 2016.

L. A. Peletier and J. Gabrielsson. Dynamics of target-mediated drug disposition. European Journal of Pharmaceutical Sciences, 38(5):445-464, 2009.

L. A. Peletier and J. Gabrielsson. Dynamics of target-mediated drug disposition: characteristic profiles and parameter identification. Journal of Pharmacokinetics and Pharmacodynamics, 39(5):429-451, 2012.

L. A. Peletier and J. Gabrielsson. Dynamics of target-mediated drug disposition: how a drug reaches its target. Computational Geosciences, 17(3):599 608, 2013.

L. A. Peletier and J. Gabrielsson. Challenges in pharmacology modelling. Journal of Dynamics and Differential Equations, 27(3-4):941-959, 2015.

G. R. Sell. Smooth linearization near a fixed point. American Journal of Mathematics, 107(5):1035-1091, 1985.

S. Sternberg. On the structure of local homeomorphisms of euclidean n-space, ii. American Journal of Mathematics, 80(3):623-631, 1958.

P. H. van der Graaf, N. Benson, and L. A. Peletier. Topics in mathematical pharmacology. Journal of Dynamics and Differential Equations, 28(34):1337-1356, 2016.

S. van Gils, M. Krupa, and P. Szmolyan. Asymptotic expansions using blowup. Zeitschrift Fur Angewandte Mathematik Und Physik, 56(3):369-397, 2005 .

\section{A Proof of Lemma 3}

We consider

$$
\begin{aligned}
& \dot{x}_{2}=-x_{2} y_{2}+z, \\
& \dot{y}_{2}=\beta-x_{2} y_{2}+z,
\end{aligned}
$$

with $z \in[0, \infty)$ a parameter. A simple phase plane analysis shows that every point in the first quadrant moves towards $y_{2} \rightarrow \infty$ with $x_{2}>0$ bounded. To study $y_{2} \rightarrow \infty$, we use a version of Poincaré compactification Meiss [2007] that follows our approach for the blowup (45). In particular, we view $\left(x_{2}, y_{2}\right)$ as coordinates on $(\bar{x}, \bar{y}, \bar{\epsilon}) \in S^{2}$ defined by

$$
x_{2}=\bar{\epsilon}^{-1 / 2} \bar{x}, \quad y_{2}=\bar{\epsilon}^{-1 / 2} \bar{y}
$$

and then study $y_{2} \rightarrow \infty$ by setting

$$
\epsilon_{3}=y_{2}^{-2}, \quad x_{3}=x_{2} y_{2}^{-1}
$$

see also (49). Notice that

$$
\epsilon_{3}=\bar{y}^{-2} \bar{\epsilon}, \quad x_{3}=\bar{y}^{-1} \bar{x}
$$

and hence the coordinates $\left(\epsilon_{3}, x_{3}\right)$ cover $\bar{y}>0$ of $(\bar{x}, \bar{y}, \bar{\epsilon}) \in S^{2}$. Inserting (70) into (69) gives $(55)_{r_{3}=0}$ within $z=$ const. By Proposition 2 (and the existence of $\mathcal{C}_{3}^{3}$ ) there exists a non-unique center manifold of $\left(\epsilon_{3}, x_{3}\right)=(0,0)$ in these coordinates with asymptotics

$$
x_{3} \approx \epsilon_{3} z
$$


for $\epsilon_{3} \rightarrow 0 . \epsilon_{3}$ decreases along this manifold. Returning to the $\left(x_{2}, y_{2}\right)$-variables using (70) gives the first result in Lemma 3. Secondly, to study $x_{2} \rightarrow \infty$ we insert

$$
\epsilon_{1}=x_{2}^{-2}, \quad y_{1}=y_{2} x_{2}^{-1}
$$

see also (48), into (69). Notice that

$$
\epsilon_{1}=\bar{x}^{-2} \bar{\epsilon}, \quad y_{1}=\bar{x}^{-1} \bar{y},
$$

and hence the coordinates $\left(\epsilon_{1}, y_{1}\right)$ cover $\bar{x}>0$ of $(\bar{x}, \bar{y}, \bar{\epsilon}) \in S^{2}$. This gives (52) within $z=$ const. and according to Proposition 1 (and the existence of $\mathcal{C}_{1}^{3}$ ) there exists a unique, attracting center manifold $\Gamma_{1}(z)$ of $\left(\epsilon_{1}, y_{1}\right)=(0,0)$ in these coordinates with asymptotics

$$
y_{1} \approx \epsilon_{1}(z+\beta),
$$

for $\epsilon_{1} \rightarrow 0 . \epsilon_{1}$ increases along this manifold. Returning to the $\left(x_{2}, y_{2}\right)$-variables then completes the proof of Lemma 3 .

\section{B Proof of Theorem 2}

The uniformity of $\Gamma_{\epsilon} \cap \mathcal{B} \rightarrow \Gamma_{0} \cap \mathcal{B}$ for $x_{0} \in(1, c]$ for any fixed $c$ follows from the proof of Theorem 1. In particular, we note that if $x_{0}=1$ then the initial condition belongs to $\mathcal{F}$ and hence $\Gamma^{2}=\emptyset$. To study $x_{0} \gg 1$ we introduce $u$ by

$$
u=x^{-1} .
$$

Then $u(0)=x_{0}^{-1} \ll 1$. Furthermore,

$$
\begin{aligned}
& u^{\prime}=-u^{2}(-y+\epsilon u z-\epsilon \alpha), \\
& y^{\prime}=-y+\epsilon \beta u(1-y)+\epsilon z u, \\
& z^{\prime}=y-\epsilon u(1+\delta) z
\end{aligned}
$$

after multiplying the right hand side by $u$ to ensure that $u=0$ is well-defined. Here $\mathcal{S}^{2}$ : $y=0,(u, z) \in[0, \infty)^{2}$ (abusing notation slightly) is normally hyperbolic (linearization gives -1 as single non-zero eigenvalue) for any $u \geq 0$. Fenichel's theory therefore applies and we obtain $\mathcal{S}_{\epsilon}^{2}$ in the following form

$$
\mathcal{S}_{\epsilon}^{2}: \quad y=\epsilon u(z+\beta+O(u \epsilon)), \quad(u, z) \in\left[0, k_{1}^{-1}\right] \times\left[0, k_{3}\right],
$$

by a simple calculation. Now, the reduced problem on $\mathcal{S}_{\epsilon}^{2}$ is described by the planar system

$$
\begin{aligned}
& \dot{u}=-u(u(\beta+O(u \epsilon))+\alpha), \\
& \dot{z}=\beta+O(u \epsilon)-\delta z
\end{aligned}
$$

where we have undone the multiplication of the right hand side by $u$. The time in (73) is therefore the slow time $t$ also used in (6). Notice that $(u, z)=\left(0, \delta^{-1} \beta\right)$ is a hyperbolic equilibrium for these equations with eigenvalues $\alpha$ and $-\delta$. To describe $\Gamma_{\epsilon}$ for $x_{0} \gg 1$ we therefore consider an initial condition $(u, z)(0)=\left(u_{0}, 0\right)$ of $(73)$ with $0<u_{0} \ll 1$. See Fig. 11 for a sketch of the phase portrait of (73) near the saddle. Clearly, the forward orbit converges to the (one-sided) stable and unstable manifold of the saddle as $u_{0} \rightarrow 0^{+}$. Now, by Sternberg [1958]; Sell [1985], there exists a $C^{1}$-linearization of $(73)$ near $(u, z)=\left(0, \delta^{-1} \beta\right)$ which is also $C^{1}$ in $\epsilon$. Therefore, although the solution spends an increasing amount of time near the saddle, the forward orbit $\Gamma_{\epsilon}$ is $O(\epsilon)$-close to $\Gamma_{0}$ in the $(u, y, z)$-variables at $u=k_{1}^{-1}$, uniformly in $u_{0}>0$. At $u=k_{1}^{-1}$ (or $x=k_{1}$ by (71)) we can change back to the $(x, y, z$ )variables. From here the analysis in Section 4 carries over. This completes the proof of the theorem. 


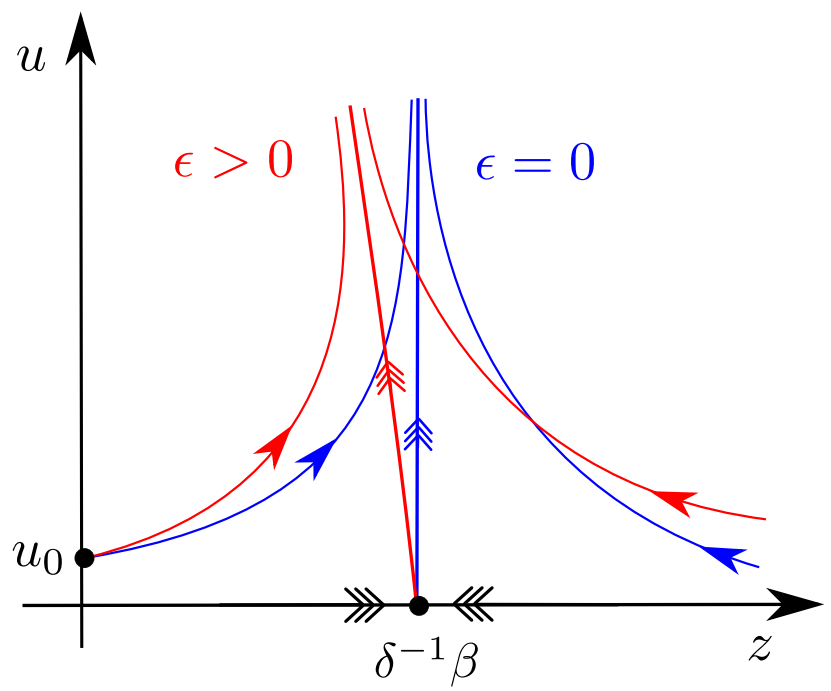

Fig. 11 Phase portrait of (73) near the saddle $(u, z)=\left(0, \delta^{-1} \beta\right)$ for $\epsilon=0$ (in blue) and $0<\epsilon \ll 1$ (in red). 